\title{
Polynomial Algorithms for Subisomorphism of nD Open Combinatorial Maps
}

\author{
Guillaume Damiand ${ }^{\mathrm{a}, *}$ Christine Solnon ${ }^{\mathrm{a}}$ Colin de la Higuera ${ }^{\mathrm{b}}$ \\ Jean-Christophe Janodet ${ }^{\mathrm{c}}$ Émilie Samuel ${ }^{\mathrm{c}}$ \\ a Université de Lyon, CNRS \\ Université Lyon 1, LIRIS, UMR5205, F-69622, France \\ ${ }^{\mathrm{b}}$ Université de Nantes, CNRS, LINA, UMR6241, F-44000, France \\ ${ }^{\mathrm{c}}$ Université de Lyon, CNRS, Université de Saint-Etienne - Jean Monnet, \\ Laboratoire Hubert Curien, UMR5516, F-42023, France
}

\begin{abstract}
Combinatorial maps describe the subdivision of objects in cells, and incidence and adjacency relations between cells, and they are widely used to model $2 \mathrm{D}$ and 3D images. However, there is no algorithm for comparing combinatorial maps, which is an important issue for image processing and analysis. In this paper, we address two basic comparison problems, i.e., map isomorphism, which involves deciding if two maps are equivalent, and submap isomorphism, which involves deciding if a copy of a pattern map may be found in a target map. We formally define these two problems for $n \mathrm{D}$ open combinatorial maps, we give polynomial time algorithms for solving them, and we illustrate their interest and feasibility for searching patterns in 2D and 3D images, as any child would aim to do when he searches Wally in Martin Handford's books.
\end{abstract}

Key words: open combinatorial maps, isomorphism and subisomorphism, pattern detection, 2D and 3D images

\footnotetext{
* Corresponding author.

Email addresses: guillaume.damiand@liris.cnrs.fr (Guillaume Damiand), christine.solnon@liris.cnrs.fr (Christine Solnon), cdlh@univ-nantes.fr (Colin de la Higuera), janodet@univ-st-etienne.fr (Jean-Christophe Janodet), emilie.samuel@univ-st-etienne.fr (Émilie Samuel).
} 


\section{Introduction}

Graphs are used in many computer graphic applications to describe images (see, for example, [CFSV07] for a review of graph-based methods for pattern recognition and computer vision). In particular, Region Adjacency Graphs (RAGs) [Ros74] model images by means of vertices - corresponding to maximal homogeneous sets of connected pixels - and edges - corresponding to adjacency relationships. RAGs are used in many image processing applications like, for example, segmentation, object extraction or comparison, and image analysis [SC84,JB93,Saa94,GMBM95,LMV01].

However, RAGs cannot model some important information contained in images. In particular, they cannot model the order in which neighbor regions are encountered when turning around some given region, as the edges incident to a vertex are not ordered. Also, RAGs cannot represent multi-adjacency. Hence, two different images may be represented by the same RAG [Kov89].

To get round this default, the RAG model has been extended. For example, [KM95] defines the dual graph structure, which is a pair of multi-graphs that represent multi-adjacency relations, and [JB98] defines ordered graphs, such that edges incident to a vertex are uniquely ordered.

Several works have proposed some solutions in 2D [Dom92,Fio96,Bru96,DBF04] and in 3D [BDDW99,Dam08] based on combinatorial maps. Indeed, combinatorial maps [Lie91] have many advantages: they are defined in any dimension; they are based on a single element called dart; they describe the subdivision of objects in cells, and incidence and adjacency relations between cells; thus they describe the topology of objects. Actually, many works have used combinatorial maps in 2D and 3D image processing algorithms [BDB97,BDD01,DR02,DD08]. However, there is no algorithm for comparing combinatorial maps, which is an important issue for image processing and image analysis.

\section{Contribution and outline of the paper}

In this paper, we address two comparison problems, i.e., map isomorphism, which involves deciding if two maps are equivalent, and submap isomorphism, which involves deciding if a copy of a pattern map may be found in a target map. We formally define these two problems for $n \mathrm{D}$ open combinatorial maps. Then we develop polynomial time algorithms for solving them, and illustrate their efficiency for searching patterns in 2D and 3D images.

Part of this work was published in $\left[\mathrm{DDLHJ}^{+} 09\right]$. Nevertheless, our previous results were limited in 2D; moreover, the material presented in Sections 4 and 
5 is totally new.

In Section 2, we recall basic definitions and notations for $n \mathrm{D}$ combinatorial maps [Lie91], and from open combinatorial maps [PABL07] thus the paper is self-contained. We use open maps to represent objects with boundaries, that allow us to model images that have blurred or undefined regions, and to define submap isomorphism problem.

In Section 3, we first extend the definition of map isomorphism of [Lie94] to open combinatorial maps and give a polynomial time algorithm for solving this problem. This algorithm is close to that of [Cor75], but we extend it to $n \mathrm{D}$ open maps. Then we tackle the submap isomorphism problem and again, we develop a polynomial time algorithm for $n \mathrm{D}$ open maps. We prove the correctness and study the complexity of both algorithms. Note that from a graph-theoretical perspective, these results imply that the subisomorphism problem for plane graphs (that is, planar graphs that are embedded in a plane) is solvable in polynomial time, whereas this problem is known intractable for general graphs. However, our algorithms are restricted to connected maps, such that there exists a path of sewn darts between every pair of darts. Thus the last part of this section is a discussion on the isomorphism problems in the case of non connected maps.

In Section 4, we introduce planar maps, that are 2D maps embedded in a plane. The goal is to design a model that is close to standard pictures: images are usually drawn on the plane, thus one region, called the external or infinite region, is distinguished and should play a particular role. Comparing planar maps ultimately returns to the problem of comparing 2D maps and then checking whether the external regions are matched or not. Therefore, we essentially use the algorithms developed in Section 3, adding new constraints. However, the knowledge of external regions allows us to optimize the efficiency of these algorithms in practice (although the worst-case complexity does not change).

Finally, in Section 5, we describe a large experimental study that proves the efficiency of our procedures in practice. We first show how to use submap isomorphism for searching for a $2 \mathrm{D}$ subimage into a database of $2 \mathrm{D}$ images. Then we tackle the searching of a 3D submesh into a database of 3D objects. These results show the interest of having generic definitions and algorithms based on $n \mathrm{D}$ open maps. Indeed, we use the same method and algorithm in different types of applications, with different dimensions, the corresponding maps being open or closed.

We conclude the paper on some related works and perspectives in Section 6 . 


\section{Combinatorial Maps}

An $n \mathrm{D}$ cellular complex is the subdivision of an $n \mathrm{D}$ object into cells of dimensions at most $n$ (0D corresponding to vertices, $1 \mathrm{D}$ to edges, $2 \mathrm{D}$ to faces, $3 \mathrm{D}$ to volumes, etc), plus incidence and adjacency relationships between cells. Cellular complexes can be modeled with combinatorial maps, which provide a generic definition based on a single basic element called dart. In Section 2.1, we recall some definitions on combinatorial maps, and in Section 2.2, we give the definition of open combinatorial maps and related notions that we use to model cellular complexes with boundaries.

\subsection{Recalls on combinatorial maps}

Combinatorial maps were originally defined for 2D [Edm60,Tut63,Cor75], then extended to 3D [AK89,Spe91] and finally to $n \mathrm{D}$ [Lie91]. Below, we briefly recall the main definitions.

Definition 1 (Combinatorial map) An nD combinatorial map, (or n-map) is a tuple $M=\left(D, \beta_{1}, \ldots, \beta_{n}\right)$ where

(1) $\mathrm{D}$ is a finite set of darts;

(2) $\beta_{1}$ is a permutation ${ }^{1}$ on $\mathrm{D}$;

(3) $\forall i: 2 \leq i \leq n, \beta_{i}$ is an involution ${ }^{2}$ on $\mathrm{D}$ with no fixed point ${ }^{3}$;

(4) $\forall i: 1 \leq i \leq n-2, \forall j: i+2 \leq j \leq n, \beta_{i} \circ \beta_{j}$ is an involution on D.

We note $\beta_{0}$ for $\beta_{1}^{-1}$, and $\beta_{i j}$ for $\beta_{j} \circ \beta_{i}$. Two darts $d$ and $d^{\prime}$ such that $d=\beta_{i}\left(d^{\prime}\right)$ are said to be $i$-sewn. Let $f$ be a function defined on a set $E$, and $X \subseteq E$, we denote $f(X)=\{f(x) \mid x \in X\}$.

Examples of 2D and 3D combinatorial maps are provided in Fig. 1 and 2.

Intuitively, $\beta_{i}$ defines adjacency relationships between cells of dimension $i$ (e.g., edges for $\beta_{1}$, faces for $\beta_{2}$, volumes for $\beta_{3}$ ). $\beta_{1}$ is a permutation (so that $\beta_{1}(d)$ may be different from $\beta_{1}^{-1}(d)$ ) whereas all other $\beta_{i}$ are involutions. Indeed, in $2 \mathrm{D}$, a face is adjacent to at most one other face whereas an edge may be adjacent to two edges of the same face: $\beta_{1}(d)$ is the dart that follows $d$ whereas $\beta_{1}^{-1}(d)=\beta_{0}(d)$ is the dart that precedes $d$. Note that $\beta_{1}$ may contain fixed points: in $2 \mathrm{D}$, they correspond to loops, i.e. faces that are bordered by a single dart.

1 A permutation on $D$ is a one-to-one mapping from $D$ to $D$.

2 An involution $f$ on $D$ is a one-to-one mapping from $D$ to $D$ such that $f=f^{-1}$.

3 A fixed point of function $f$ is an element $e$ such that $f(e)=e$. 


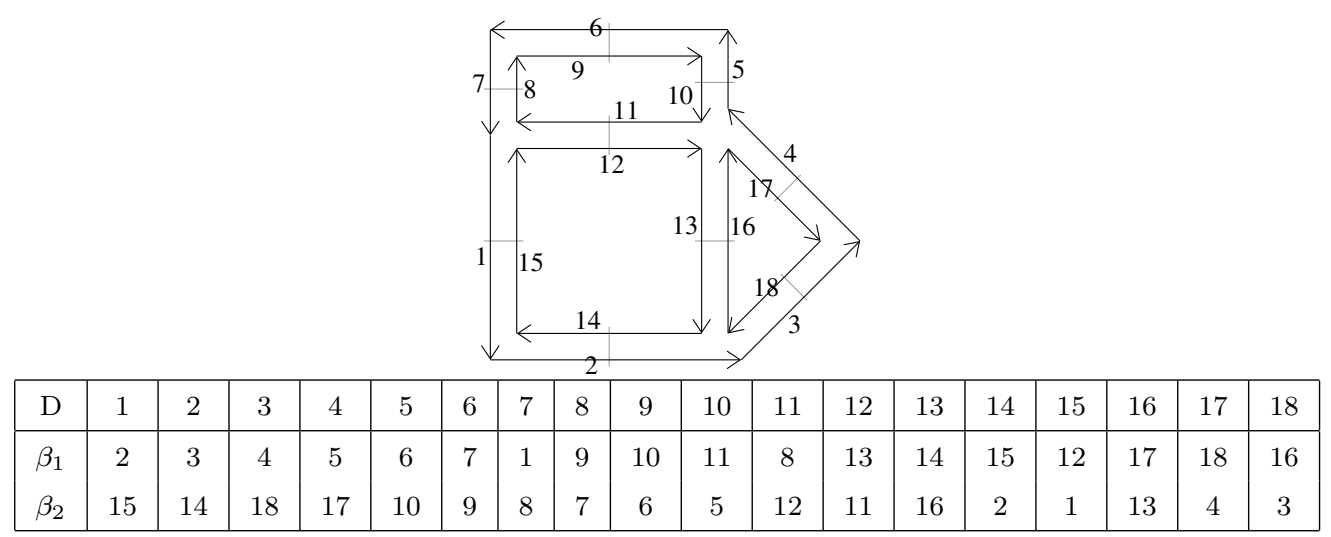

Fig. 1. An example of 2D combinatorial map. Darts are represented by black arrows. Two 1-sewn darts are drawn consecutively, and two 2-sewn darts are concurrently drawn, in reverse orientation, with little a grey segment between them.

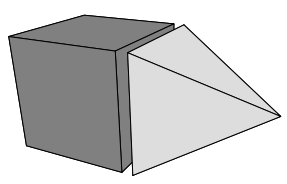

(a)
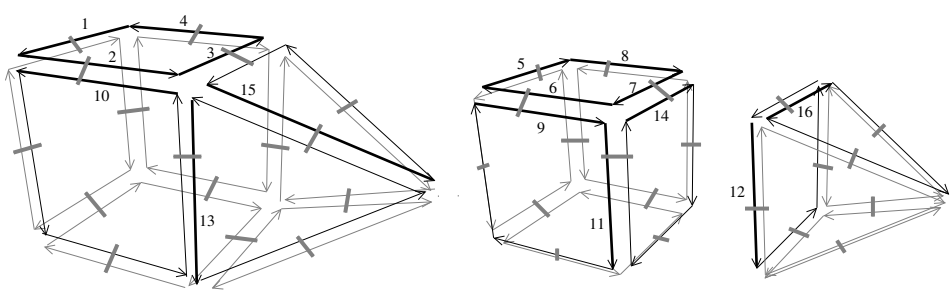

(b)

\begin{tabular}{|c|c|c|c|c|c|c|c|c|c|c|c|c|c|c|c|c|}
\hline $\mathrm{D}$ & 1 & 2 & 3 & 4 & 5 & 6 & 7 & 8 & 9 & 10 & 11 & 12 & 13 & 14 & 15 & $\ldots$ \\
\hline$\beta_{1}$ & 2 & 3 & 4 & 1 & 8 & 5 & 6 & 7 & 11 & $\ldots$ & $\ldots$ & $\ldots$ & $\ldots$ & $\ldots$ & $\ldots$ & $\ldots$ \\
$\beta_{2}$ & $\ldots$ & 10 & $\ldots$ & $\ldots$ & $\ldots$ & 9 & 14 & $\ldots$ & 6 & 2 & $\ldots$ & $\ldots$ & $\ldots$ & 7 & $\ldots$ & $\ldots$ \\
$\beta_{3}$ & 5 & 6 & 7 & 8 & 1 & 2 & 3 & 4 & 10 & 9 & $\ldots$ & $\ldots$ & $\ldots$ & $\ldots$ & $\ldots$ & $\ldots$ \\
\hline
\end{tabular}

Fig. 2. An example of a 3D combinatorial map. (a) A 3D object. (b) The corresponding 3D combinatorial map (external volume on the left; interior on the middle and the right). The graphical convention is the same as in $2 \mathrm{D} . \beta_{3}$ is not drawn, but (partially) given in the array.

In combinatorial maps, cells are defined by means of orbits. Given a set $E$, a set $\left\{p_{1}, \ldots, p_{j}\right\}$ of permutations on $E$ and an element $e \in E$, the orbit $\left\langle p_{1}, \ldots, p_{j}\right\rangle(e)$ is the set of all elements of $E$ that can be reached from $e$ by composing any $p_{1}, \ldots, p_{j}$ and their inverses $p_{1}^{-1}, \ldots, p_{j}^{-1}$.

The link between cells and orbits is given in Def. 2 .

Definition 2 (i-cell) Let $M$ be an n-map, and $d$ a dart.

- The 0-cell incident to $d$ is $\left\langle\beta_{02}, \ldots, \beta_{0 n}\right\rangle(d)$;

- $\forall i$ : $1 \leq i \leq n$, the $i$-cell incident to $d$ is $\left\langle\beta_{1}, \ldots, \beta_{i-1}, \beta_{i+1}, \ldots, \beta_{n}\right\rangle(d)$.

Hence, a cell is a set of darts.

- A 0-cell corresponds to a vertex and is a set of darts that share a same origin. 
In $2 \mathrm{D}$, a 0 -cell is obtained by the orbit $\left\langle\beta_{02}\right\rangle$ (e.g., $\left\langle\beta_{02}\right\rangle(8)=\{1,8,12\}$ in Fig. 1). In $3 \mathrm{D}$, it is obtained by the orbit $\left\langle\beta_{02}, \beta_{03}\right\rangle$ (e.g., $\left\langle\beta_{02}, \beta_{03}\right\rangle(3)=$ $\{3,6,10,11,12,13,14,15,16\}$ in Fig. 2);

- A 1-cell corresponds to an edge and is a set of darts that share their endpoints. In $2 \mathrm{D}$, it is obtained by the orbit $\left\langle\beta_{2}\right\rangle$ (e.g., $\left\langle\beta_{2}\right\rangle(8)=\{7,8\}$ in Fig. 1). In $3 \mathrm{D}$, it is obtained by the orbit $\left\langle\beta_{2}, \beta_{3}\right\rangle$ (e.g., $\left\langle\beta_{2}, \beta_{3}\right\rangle(2)=\{2,6,9,10\}$ in Fig. 2);

- A 2-cell corresponds to a face. In $2 \mathrm{D}$, it is obtained by the orbit $\left\langle\beta_{1}\right\rangle$ (e.g., $\left\langle\beta_{1}\right\rangle(8)=\{8,9,10,11\}$ in Fig. 1). In 3D, it is obtained by the orbit $\left\langle\beta_{1}, \beta_{3}\right\rangle$ (e.g., $\left\langle\beta_{1}, \beta_{3}\right\rangle(1)=\{1,2,3,4,5,6,7,8\}$ in Fig. 2 );

- A 3-cell corresponds to a volume and is obtained in 3D by the orbit $\left\langle\beta_{1}, \beta_{2}\right\rangle$ (e.g., $\left\langle\beta_{1}, \beta_{2}\right\rangle(5)$ contains the 24 darts of the internal cube in Fig. 2).

We can define the incidence and adjacency relations between cells:

- Two cells are incident if their intersection is not empty. E.g., edge $\left\langle\beta_{2}\right\rangle(7)=$ $\{7,8\}$ is incident to face $\left\langle\beta_{1}\right\rangle(8)=\{8,9,10,11\}$ in Fig. 1 .

- Two $i$-cells are adjacent if there is an $(i-1)$-cell incident to both $i$-cells. E.g., faces $\left\langle\beta_{1}\right\rangle(8)$ and $\left\langle\beta_{1}\right\rangle(15)$ are adjacent because they are both incident to edge $\left\langle\beta_{2}\right\rangle(11)$ in Fig. 1.

\subsection{Open combinatorial maps}

In [Lie91], Lienhardt has defined generalized maps, which can be used to model open or closed objects, thus allowing one to model objects with boundaries. A preliminary report [PABL07] extended the definition of $n \mathrm{D}$ combinatorial maps to open $n \mathrm{D}$ combinatorial maps.

Open maps may contain free darts, i.e., darts that are not linked with other darts for some dimensions. In generalized maps [Lie91], a dart is $i$-free whenever it is $i$-sewn with itself (i.e., $\left.\beta_{i}(d)=d\right)$. However, in combinatorial maps, $\beta_{1}$ may contain fixed points (in case of loops), thus such a trick is impossible to re-use. Therefore, to denote that a dart is 1 -free, a new element $\epsilon$ is considered in addition to the set of darts, and darts can be 1-sewn with $\epsilon$. To make things similar in every dimensions, we use the same principle for $i$-free darts, with $i>1$. Thus, a dart $d$ is $i$-free, for $i \in\{0, \ldots, n\}$, if $\beta_{i}(d)=\epsilon$.

However, if some darts are $i$-sewn with $\epsilon$, then $\beta_{i}$ is no longer a permutation or an involution on $D$, but a partial permutation or partial involution such that only a subset $X \subseteq D$ of darts is linked to another subset of darts of $D$ whereas other darts are $i$-free. We formally define a partial permutation as well as its inverse as follows.

Definition 3 (Partial permutation) Let $E$ be a set, $X \subseteq E$ a subset of $E$, 
and $f: X \rightarrow E$ an injection. The partial permutation $p$ on $E$ induced by $f$ is defined by

(1) $p(\epsilon)=\epsilon$;

(2) $\forall e \in E$, if $e \in X$ then $p(e)=f(e)$, otherwise $p(e)=\epsilon$.

The inverse $p^{-1}$ of this partial permutation is also a partial permutation and is defined by

(1) $p^{-1}(\epsilon)=\epsilon$;

(2) $\forall e \in E$, if $e \in f(X)$, then $p^{-1}(e)=f^{-1}(e)$, otherwise $p^{-1}(e)=\epsilon$.

In other words, a partial permutation of $E$ is a bijection between two subsets $F$ and $G$ of $E$ such that the elements of $E$ that do not belong to $F$ or $G$ are linked to $\epsilon$.

A partial involution is a specific partial permutation, satisfying the condition $f(f(e))=e$ for any element $e$ such that $f(e)=\epsilon$.

Definition 4 (Partial involution) A partial involution $f$ on $E$ is a partial permutation on $E$ such that: $\forall e \in E, f(e) \neq \epsilon \Rightarrow f(f(e))=e$.

Since a partial involution $f$ is a partial permutation (with an additional property), its inverse is defined in Def. 3, and we have $f^{-1}=f$. Moreover, we can verify that, given two partial permutations $f$ and $g, f \circ g$ is a partial permutation, and $(f \circ g)^{-1}=g^{-1} \circ f^{-1}$.

We can now define open combinatorial maps.

Definition 5 (Open combinatorial map) An open $n D$ combinatorial map (or open $n$-map) is a tuple $M=\left(D, \beta_{1}, \ldots, \beta_{n}\right)$ where

(1) $D$ is a finite set of darts;

(2) $\beta_{1}$ is a partial permutation on $D$;

(3) $\forall i: 2 \leq i \leq n, \beta_{i}$ is a partial involution on $D$ with no fixed point;

(4) $\forall i: 0 \leq i \leq n-2, \forall j: 3 \leq j \leq n, i+2 \leq j, \beta_{i j}$ is a partial involution.

Open $n$-maps differ from $n$-maps on three points: (1) $\beta_{1}$ is a partial permutation instead of a permutation; (2) other $\beta_{i}$ are partial involutions; (3) Condition 4 is modified such that $\beta_{i j}$ is a partial involution, and this condition must also be satisfied for $i=0$. An example of open 2-map is represented in Fig. 3.

The first two differences directly come from the fact that we can have free darts. For the third difference, we need to add the condition on $\beta_{0 j}$ because $\beta_{1 j}$ is a partial involution does not imply that $\beta_{0 j}$ is a partial involution (contrary to the original definition of combinatorial maps where $\beta_{1 j}$ is an involution 


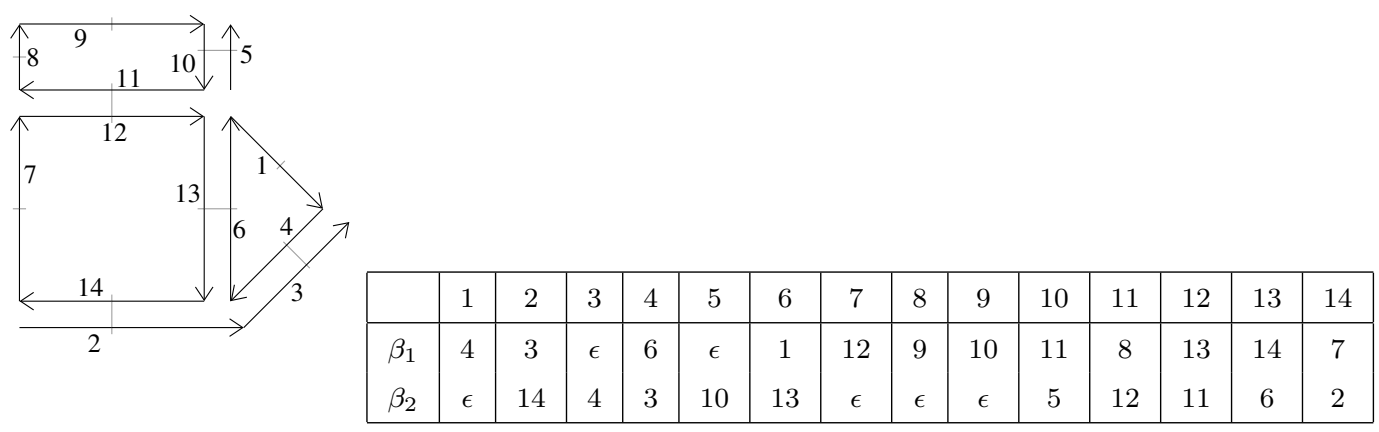

Fig. 3. Open combinatorial map example. Darts 3 and 5 are 1 -free, and darts 1, 7, 8 and 9 are 2 -free.

implies that $\beta_{0 j}$ is an involution).

A combinatorial map is said to be $i$-open (resp. $i$-closed), for $i \in\{1, \ldots, n\}$, if it contains at least one $i$-free dart (resp. no $i$-free dart). The map is said to be open (resp. closed) if it is $i$-open for at least one $i \in\{1, \ldots, n\}$ (resp. $i$-closed for all $i \in\{1, \ldots, n\})$. When nothing is specified, a combinatorial map may be either open or closed, and thus it is defined with respect to Def. 5 .

From a mathematical standpoint, any combinatorial map denotes an $n \mathrm{D}$ cellular quasi-manifold [Lie94]. As every combinatorial map can easily be converted into a generalized map, this semantics still holds for our definition of open combinatorial map.

Now let us tackle the definition of cells.

The definition of orbits given in Section 2.1 is still valid for open combinatorial maps, since it uses "the set of all elements of E that can be reached...", which avoid to have $\epsilon$ in any orbit (since $\epsilon \notin E$ by partial permutation definition). For this reason, the Def. 2 of $i$-cells given for closed maps, is still valid for $i>1$.

For instance, in the 2-map displayed in Fig. 3, the edge incident to dart 1 is $\left\langle\beta_{2}\right\rangle(1)=\{1\}$ (since dart 1 is 2 -free) while the edge incident to dart 11 is $\left\langle\beta_{2}\right\rangle(11)=\{11,12\}$. The face incident to dart 2 is $\left\langle\beta_{1}\right\rangle(2)=\{2,3\}$.

However, we need to modify the definition of vertices (0-cells in Def. 6).

Definition 6 (0-cell) Let $M=\left(D, \beta_{1}, \ldots, \beta_{n}\right)$ be an $n$-map, and $d \in D$. The 0 -cell incident to $d$ is the set $\left\langle\beta_{02}, \ldots, \beta_{0 n},\left\{\beta_{i j} \mid \forall i, j: 2 \leq i<j \leq n\right\}\right\rangle(b)$.

The difference with the previous definition of 0 -cells is that we need to add $\beta_{i j}$ for all $2 \leq i<j \leq n$ in order not to miss some darts that cannot be reached due to some free darts. Let us consider, for example, the 0-cell incident to 3 in the closed 3-map of Fig. 2, that is, $\left\langle\beta_{02}, \beta_{03}\right\rangle(3)=\{3,6,10,11,12,13,14,15,16\}$. Some darts in this orbit are reached from 3 by composing some permutations, 
e.g., $\beta_{02}\left(\beta_{03}(3)\right)=11$. Let us now consider the 3-map obtained by removing darts 2 and 4 (i.e., dart 3 is 0 -free and 1-free). In this case, we have to use $\beta_{23}$ and $\beta_{23}^{-1}$ to reach other darts of the vertex incident to dart $3\left(\right.$ e.g., $\left.\beta_{23}(3)=11\right)$, since $\beta_{02}(3)=\beta_{03}(3)=\beta_{21}(3)=\beta_{31}(3)=\epsilon$.

In the example of the 2-map displayed in Fig. 3, the vertex incident to dart 12 is $\left\langle\beta_{02}\right\rangle(12)=\{8,12\}$.

\section{Map and Submap Isomorphism}

(Sub)map isomorphism allows one to compare maps. In Section 3.1, we define map isomorphism - which allows one to decide the equivalence of two mapsand give a polynomial time algorithm for solving this problem. In Section 3.2, we consider submap isomorphism - which allows one to decide of the inclusion of a pattern map into a target map - and propose a polynomial time algorithm for solving this problem too.

In Sections 3.1 and 3.2, we only consider connected maps in which there exists a path of sewn darts between every pair of darts. Formally:

Definition 7 (Path) Let $M=\left(D, \beta_{1}, \ldots, \beta_{n}\right)$ be a combinatorial map. A sequence of darts $\left(d_{1}, \ldots, d_{k}\right)$ is a path between $d_{1}$ and $d_{k}$ if $\forall i: 1 \leq i<k, \exists j_{i} \in\{0,1, \ldots, n\}, d_{i+1}=\beta_{j_{i}}\left(d_{i}\right)$.

Definition 8 (Connected map) A combinatorial map $M=\left(D, \beta_{1}, \ldots, \beta_{n}\right)$ is connected if $\forall d \in D, \forall d^{\prime} \in D$, there exists a path between $d$ and $d^{\prime}$.

The extension of our work to non connected maps is discussed in Section 3.3.

\subsection{Map isomorphism}

Lienhardt [Lie94] has defined isomorphism between two closed combinatorial maps. We extend this definition to open combinatorial maps as follows.

Definition 9 (Map isomorphism) Two n-maps $M=\left(D, \beta_{1}, \ldots, \beta_{n}\right)$ and $M^{\prime}=\left(D^{\prime}, \beta_{1}^{\prime}, \ldots, \beta_{n}^{\prime}\right)$ are isomorphic if there exists a bijection $f: D \cup\{\epsilon\} \rightarrow$ $D^{\prime} \cup\{\epsilon\}$, called isomorphism function, such that $f(\epsilon)=\epsilon$ and $\forall d \in D, \forall i$ : $1 \leq i \leq n, f\left(\beta_{i}(d)\right)=\beta_{i}^{\prime}(f(d))$.

The only difference with the definition of isomorphism between closed $n$-maps is that we have added that $f(\epsilon)=\epsilon$. Indeed, if a dart is $i$-sewn with $\epsilon$, then the dart matched to it by $f$ must also be $i$-sewn with $\epsilon$. 
As already mentioned in [Cor75] for 2D maps, an algorithm for deciding of the isomorphism of two connected maps can easily be derived from this definition. Indeed, consider Algorithm 1. We first fix a dart $d_{0} \in D$ and, for every dart $d_{0}^{\prime} \in D^{\prime}$, we call Algorithm 2 to build a candidate matching function $f$ and then we check whether $f$ actually is an isomorphism function. Algorithm 2 basically performs a traversal of $M$, starting from $d_{0}$ and using the $\beta_{i}$ functions to discover new darts from darts that have already been discovered: initially, $f\left[d_{0}\right]$ is set to $d_{0}^{\prime}$ whereas $f[d]$ is set to nil for all other darts, thus stating that $d$ has not yet been discovered; then, each time a dart $d \in D$ is discovered, from another dart $d_{k} \in D$ such that $d$ is $i$-sewn with $d_{k}$, then $f[d]$ is set to the dart $d^{\prime} \in D^{\prime}$ which is $i$-sewn with $f\left[d_{k}\right]$.
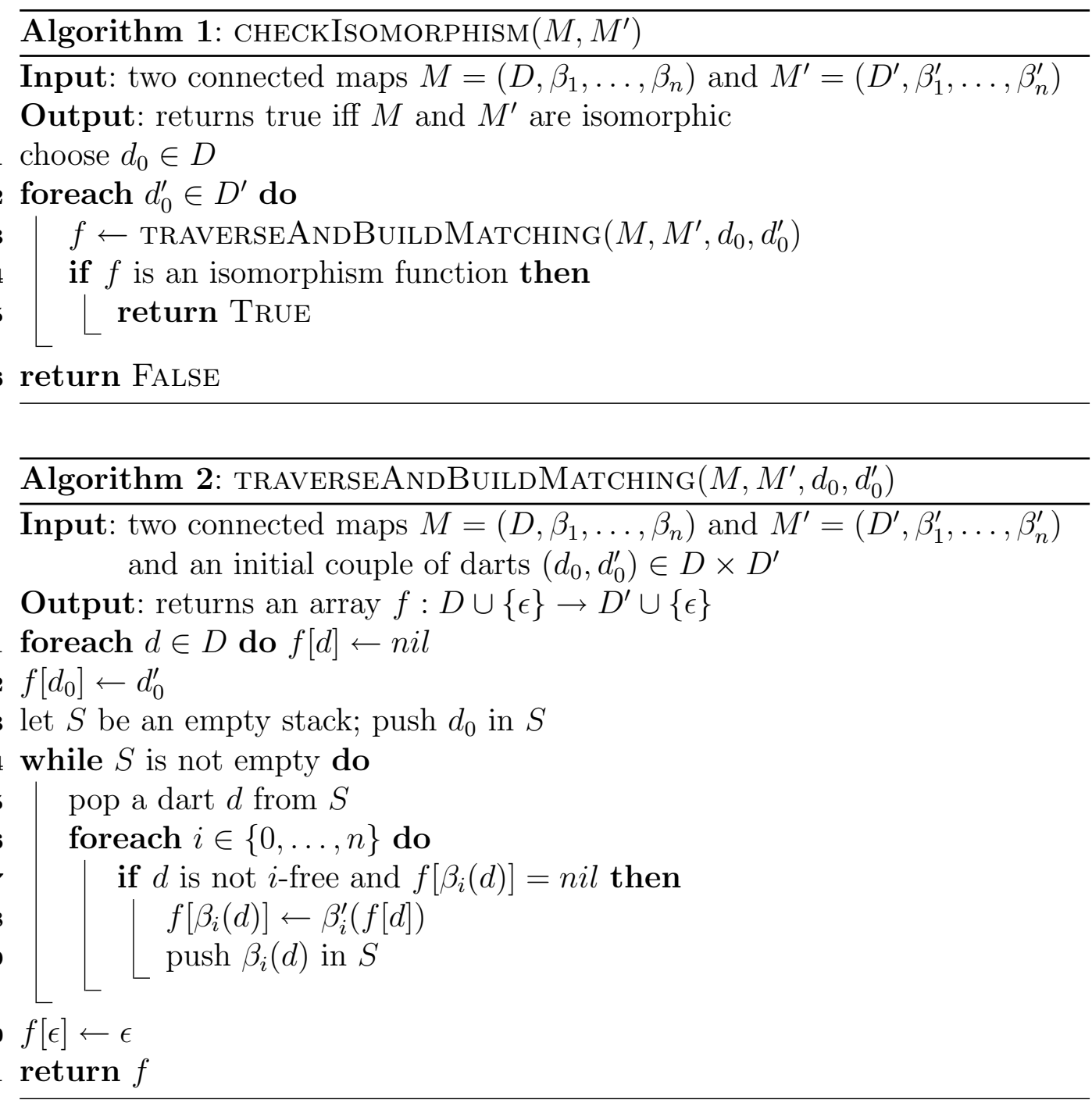

We get the following result:

Theorem 10 Two nD connected maps $M$ and $M^{\prime}$ are isomorphic iff $\operatorname{CHECKISOMORPhism}\left(M, M^{\prime}\right)$ returns true. 
PROOF. This corresponds to proving that Algorithm 1 is correct.

$(\Leftarrow)$ If $\operatorname{CHeCkIsomorphism}\left(M, M^{\prime}\right)$ returns true, then $M$ and $M^{\prime}$ are isomorphic since true is returned only if the test in line 4 succeeds.

$(\Rightarrow)$ Let us suppose that $M$ and $M^{\prime}$ are isomorphic, so that there exists an isomorphism function $\varphi: D \rightarrow D^{\prime}$, and let us show that CHECKIsOMOR$\operatorname{Phism}\left(M, M^{\prime}\right)$ returns true. Let $d_{0} \in D$ be the dart chosen at line 1 of Algorithm 1. As the loop lines 2-5 iterates on every dart $d_{0}^{\prime} \in D^{\prime}$, there exists an iteration of this loop for which $d_{0}^{\prime}=\varphi\left(d_{0}\right)$. Let us show that for this iteration TRAVERSEANDBuildMATChing $\left(M, M^{\prime}, d_{0}, d_{0}^{\prime}\right)$ returns the array $f$ such that $\forall d \in D, f[d]=\varphi(d)$ so that true is returned line 5 of Algorithm 1:

- Claim 1: When pushing a dart d in $S, f[d]=\varphi(d)$. By induction. The claim holds for the push of line 3 as $f\left[d_{0}\right]$ is set to $d_{0}^{\prime}=\varphi\left(d_{0}\right)$ at line 2 . The claim also holds for the push at line 9 as $(1) f\left[\beta_{i}(d)\right]$ is set to $\beta_{i}^{\prime}(f[d])$ in line 8 and (2) $f[d]=\varphi(d)$ (by induction hypothesis) and (3) $\varphi(d)=d^{\prime} \Rightarrow \varphi\left(\beta_{i}(d)\right)=$ $\beta_{i}^{\prime}\left(d^{\prime}\right)$ (by definition of an isomorphism function).

- Claim 2: Every dart $d \in D$ is pushed once in $S$. Indeed, $M$ is connected, so that there exists at least one path of sewn darts $\left(d_{0}, \ldots, d_{n}\right)$ such that $d_{n}=d$. Therefore, each time a dart $d_{i}$ of this path is popped from $S$ (line 5 ), $d_{i+1}$ is pushed in $S$ (line 9) if it has not been pushed before (through another path).

The time complexity of Algorithm 1 is $\mathcal{O}\left(n \cdot|D| \cdot\left|D^{\prime}\right|\right)$

Let us first show that the time complexity of Algorithm 2 is $\mathcal{O}(n \cdot|D|)$. Indeed, the for loop (lines 6-9) is iterated $n$ times, and the while loop (lines 4-9) is iterated $|D|$ times as (1) exactly one dart $d$ is removed from the stack $S$ at each iteration, and (2) each dart $d \in D$ enters $S$ at most once (it enters $S$ only if $f[d]=$ nil, and before entering $S, f[d]$ is set to a dart of $D^{\prime}$ ). Let us then note that the test from line 4 of Algorithm 1 may be performed in $\mathcal{O}(n \cdot|D|)$. As Algorithm 2 and the test of line 4 are performed at most $\left|D^{\prime}\right|$ times (once for each dart of $M^{\prime}$ ), the overall time complexity of Algorithm 1 is $\mathcal{O}\left(n \cdot|D| \cdot\left|D^{\prime}\right|\right)$.

Note that Algorithm 1 may be optimized, without changing its worst-case complexity. In particular, we could detect failure while building matchings by checking between lines 7 and 8 of Algorithm 2 that there does not exist a dart $d_{j} \in D$ that has already been matched to $\beta_{i}^{\prime}(f[d])$ : if this is the case, one can stop the current traversal as $f$ will not be a bijection. 


\subsection{Submap isomorphism}

We now tackle the problem of submap isomorphism, the goal of which is to decide if there exists a copy of a pattern map in a target map.

Basically, a submap is obtained from a map by keeping only a subset $X$ of its darts, and updating $\beta_{i}$ functions so that every dart of $X$ that is $i$-sewn with a dart that does not belong to $X$ becomes $i$-free.

Definition 11 (Submap) Given a combinatorial map $M=\left(D, \beta_{1}, \ldots, \beta_{n}\right)$ and a subset of darts $X \subseteq D$, the submap of $M$ induced by $X$, denoted $M_{\downarrow X}$ is the combinatorial map $\left(X, \gamma_{1}, \ldots, \gamma_{n}\right)$ such that:

$\forall d \in X, \forall i: 1 \leq i \leq n$, if $\beta_{i}(d) \notin X$ then $\gamma_{i}(d)=\epsilon$; else $\gamma_{i}(d)=\beta_{i}(d)$.

Note that, depending on $X$, the submap $M_{\downarrow X}$ may not satisfy condition 4 of Def. 5 so that it may not be a combinatorial map. Hence, we shall verify, when using Def. 11, that the subset $X$ is such that $M_{\downarrow X}$ satisfies this condition.

We can now define submap isomorphism as follows.

Definition 12 (Submap isomorphism) Let $M=\left(D, \beta_{1}, \ldots, \beta_{n}\right)$ and $M^{\prime}=$ $\left(D^{\prime}, \beta_{1}^{\prime}, \ldots, \beta_{n}^{\prime}\right)$ be two $n$-maps. $M$ is isomorphic to a submap of $M^{\prime}$ if there exists a subset of darts $X \subseteq D^{\prime}$ such that $M_{\downarrow X}^{\prime}$ is isomorphic to $M$.

The subset $X$ obviously satisfies condition 4 of Def. 5 since if $M_{\downarrow X}^{\prime}$ is isomorphic to $M$, as $M$ is a valid combinatorial map, its darts satisfy this condition.

The existence of a submap isomorphism implies the existence of a subisomorphism function which matches every dart of the pattern map to a different dart of the target map, so that pattern darts that are $i$-sewn are matched to target darts that are also $i$-sewn, like an isomorphism function. However, when a pattern dart $d$ is $i$-free the target dart matched to $d$ must be either $i$-free, or it must be $i$-sewn with a target dart which is not matched to another pattern dart (see example in Fig. 4). This is more formally stated in Theorem 13.

Theorem $13 M$ is isomorphic to a submap of $M^{\prime}$ iff there exists an injection $f: D \cup\{\epsilon\} \rightarrow D^{\prime} \cup\{\epsilon\}$, called a subisomorphism function, such that:

(1) $f(\epsilon)=\epsilon$ and

(2) $\forall d \in D, \forall i: 1 \leq i \leq n$,

- if $d$ is not $i$-free, then $\beta_{i}^{\prime}(f(d))=f\left(\beta_{i}(d)\right)$;

- otherwise, either $f(d)$ is $i$-free, or $\left.\forall d_{k} \in D, f\left(d_{k}\right) \neq \beta_{i}^{\prime}(f(d))\right)$.

\section{PROOF.}




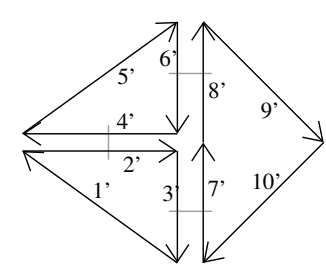

(a) $M$

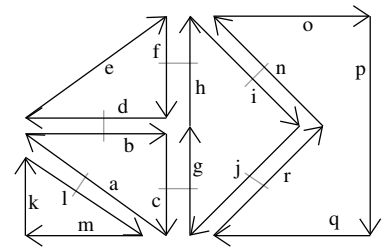

(b) $M^{\prime}$

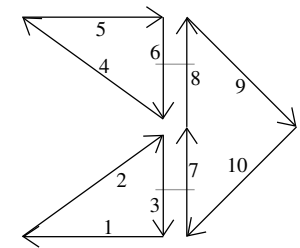

(c) $M^{\prime \prime}$

Fig. 4. Submap isomorphism example. $M$ is a submap of $M^{\prime}$ as it is obtained from $M^{\prime}$ by deleting darts $k$ to $r . M^{\prime \prime}$ is not isomorphic to a submap of $M^{\prime}$ as the injection $f: X \rightarrow D$ that respectively matches darts 1 to 10 to darts $a$ to $j$ does not verify Theorem 13: for example, dart 4 is 2-free and it is matched to dart $d$ which is 2-sewn with a dart $(b)$ which is itself matched (i.e., $f^{-1}(b)=2$ ).

$(\Rightarrow)$ If $M$ is isomorphic to a submap of $M^{\prime}$, then there exists a subset $X \subseteq$ $D^{\prime}$ such that $M$ is isomorphic to $M_{\downarrow X}^{\prime}$. By Def. 9, there exists a bijection $f: D \cup\{\epsilon\} \rightarrow X \cup\{\epsilon\}$ such that $f(\epsilon)=\epsilon$ and $\forall d \in D, \forall i: 1 \leq i \leq$ $n, f\left(\beta_{i}(d)\right)=\beta_{i}^{\prime}(f(d))$. Let us define function $g: D \cup\{\epsilon\} \rightarrow D^{\prime} \cup\{\epsilon\}$ such that $\forall d \in D, g(d)=f(d)$ and $g(\epsilon)=\epsilon$, and let us show that $g$ is a subisomorphism function. Obviously, $\forall i: 1 \leq i \leq n$, if $d$ is not $i$-free, then $\beta_{i}^{\prime}(g(d))=g\left(\beta_{i}(d)\right)$. The key point is to show that if $d$ is $i$-free, then either $g(d)$ is $i$-free or $\forall d_{k} \in$ $\left.D, f\left(d_{k}\right) \neq \beta_{i}^{\prime}(f(d))\right)$. Let us suppose that $g(d)$ is not $i$-free and there exists $d_{k} \in D$ such that $g\left(d_{k}\right)=\beta_{i}^{\prime}(g(d))$. As $f$ is an isomorphism function and $g$ is the restriction of $f$ to $X$, we have $g\left(d_{k}\right)=\beta_{i}^{\prime}(g(d))=g\left(\beta_{i}(d)\right)$ so that $d_{k}=\beta_{i}(d)$ which is in contradiction with the fact that $d$ is $i$-free.

$(\Leftarrow)$ Let us suppose that there exists a subisomorphism function $f: D \cup\{\epsilon\} \rightarrow$ $D^{\prime} \cup\{\epsilon\}$. We must show that $M$ is isomorphic to a submap of $M^{\prime}$, i.e., that there exists a subset $X \subseteq D$ such that $M$ is isomorphic to $M_{\downarrow X}^{\prime}$. Obviously, we define $X=\{f(d) \mid d \in D\}$ and function $g: D \rightarrow X$ such that $g(\epsilon)=\epsilon$ and $\forall d \in D, g(d)=f(d)$.

Algorithm 3 determines if there is a submap isomorphism between two connected maps. It is based on the same principle as Algorithm 1; the only difference is the test of line 4 , which succeeds if $f$ is a subisomorphism function instead of an isomorphism function.

Correctness proofs and evidences given for isomorphism are still valid: we solve the submap isomorphism problem with the same method as before, except that function $f$ is now an injection instead of a bijection.

The time complexity of this algorithm is $\mathcal{O}\left(n \cdot|D| \cdot\left|D^{\prime}\right|\right)$ as TRAVERSEANDBuildMatching is called at most $\left|D^{\prime}\right|$ times and its complexity is $\mathcal{O}(n \cdot|D|)$. Note that the subisomorphism test may be done in linear time.

Also, one may optimize Algorithm 3 in a similar way as proposed for Al- 


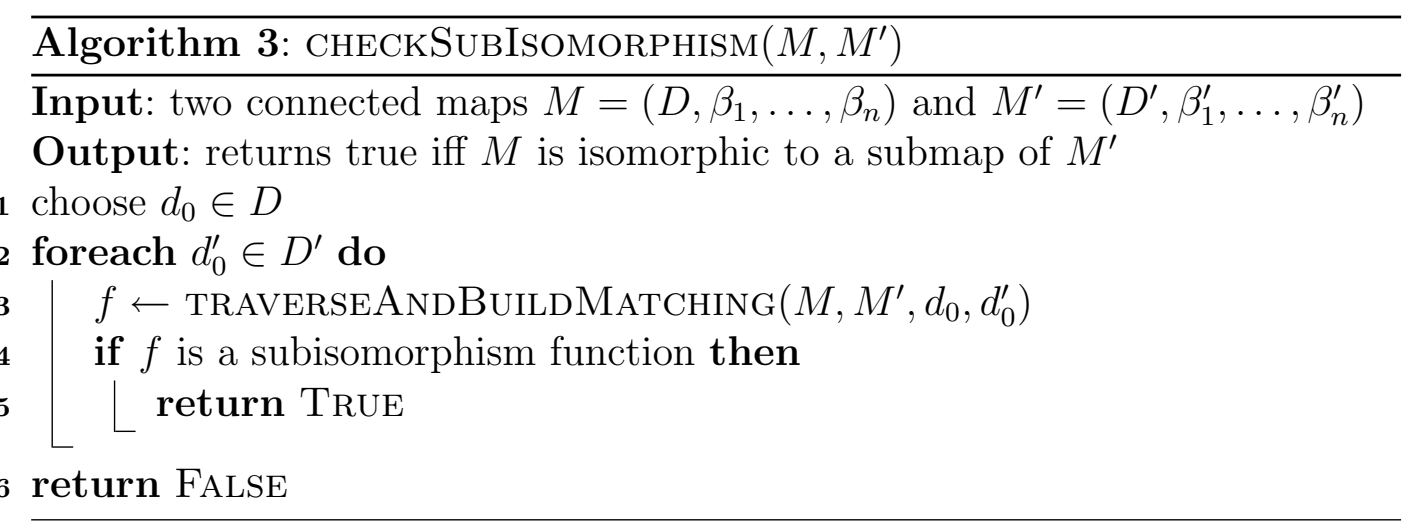

gorithm 1, without changing its worst-case complexity, by checking between lines 7 and 8 of Algorithm 2 that there does not exist a dart $d_{j} \in D$ that has already been matched to $\beta_{i}^{\prime}(f[d])$ : if this is the case, one can stop the current traversal as $f$ will not be an injection.

\section{3 (Sub)map isomorphism of non connected maps}

The algorithms given in Sections 3.1 and 3.2 have been designed for connected maps, such that there exists a path between any pair of darts. This condition is necessary to ensure that all darts of $M$ are discovered during the traversal, whatever the initial dart $d_{0}$ is. In this section, we discuss the extension of these algorithms to non connected maps.

Isomorphism of non connected maps. This case is solvable in polynomial time. Indeed, each non connected map may be decomposed into a set of connected maps. Let $M=\left\{M_{1}, \ldots, M_{k}\right\}$ and $M^{\prime}=\left\{M_{1}^{\prime}, \ldots, M_{k^{\prime}}^{\prime}\right\}$ be the sets of connected maps that respectively compose $M$ and $M^{\prime}$. Obviously, $M$ and $M^{\prime}$ are not isomorphic if $k \neq k^{\prime}$. If $k=k^{\prime}$, one may decide of the isomorphism of $M$ and $M^{\prime}$ as follows:

(1) Build the bipartite graph $G=\left(M, M^{\prime}, E\right)$ such that $E$ associates an edge between connected maps $\left(M_{i}, M_{j}^{\prime}\right) \in M \times M^{\prime}$ iff $M_{i}$ and $M_{j}^{\prime}$ are isomorphic;

(2) Decide if there exists a perfect matching ${ }^{4}$, thus ensuring that, for each connected map $M_{i} \in M$, there exists a different connected map $M_{j}^{\prime} \in M^{\prime}$ such that $M_{i}$ and $M_{j}^{\prime}$ are isomorphic.

$\overline{4}$ A matching of a bipartite graph $G=\left(V_{1}, V_{2}, E\right)$ is a subset of edges $E^{\prime} \subseteq E$ such that each vertex is the endpoint of at most one edge. A matching $E^{\prime}$ is perfect if each vertex is covered ones. 
Step (1) may be done in $\mathcal{O}\left(n \cdot|D| \cdot\left|D^{\prime}\right|\right)$. Step (2) may be done in $\mathcal{O}\left(k^{2}\right)$, where $k$ is the number of connected maps that compose $M$. Indeed, all connected components of the bipartite graph $G$ are complete bipartite graphs (as the isomorphism relationship is transitive). As a consequence, $G$ has a perfect matching iff, for each connected component $X$ of $G$, the number of nodes of $M$ in $X$ is equal to the number of nodes of $M^{\prime}$ in $X$ (or, in other words, the number of isomorphic components is the same in $M$ and $M^{\prime}$ ). Hence, step (2) may be achieved by a simple traversal of the bipartite graph. Note that relative positions of the different components do not matter and cannot be detected. Indeed a triangle inside a complex map would be isomorphic to a triangle beside the same map.

Subisomorphism of non connected maps. If the pattern map $M$ is connected whereas the target map $M^{\prime}$ is non connected, then subisomorphism may be decided by checking that $M$ if a submap of at least one connected map that composes $M^{\prime}$, and Algorithm 3 can directly be used.

Let us now focus on the case where the pattern map $M$ is non connected and let us show that it is $N P$-complete. Indeed, it may be used to solve $N P$-complete tiling problems such as, for example, the two-dimensional strip packing problem. An instance of this problem is defined by a set of $n$ rectangular items, each having a given integer width $w_{i}$ and height $h_{i}$, and a bin of integer width $W$ and height $H$. The goal is to decide if it is possible to pack all items in the bin without overlapping. The basic idea of the reduction of this problem to a submap isomorphism problem is to define a first non connected pattern map that associates a connected component with each item, and a second connected target map corresponding to the bin, and then look for a submap isomorphism. The tricky point comes from the fact that the images of different connected components of the pattern map cannot be sewn in the target map (by definition of submap isomorphism). Therefore, we modify the sizes of the maps associated with the items and the bin. More precisely, the pattern and target maps are defined as follows:

- The pattern map is a non connected map composed of $k$ connected components: for each item of width $w_{i}$ and height $h_{i}$, we define a connected component composed of $\left(2 * w_{i}-1\right) \times\left(2 * h_{i}-1\right)$ square faces that are 2 -sewn in order to form a rectangle;

- The target map is a connected map composed of $(2 * W-1) \times(2 * H-1)$ square faces that are 2 -sewn to form a rectangle.

One can easily deduce a solution to the strip packing problem from the solution to the submap isomorphism problem, as illustrated in Fig. 5. Since each instance of strip packing can be transformed into an instance of submap isomorphism problem, and since each solution of submap problem allows to find 


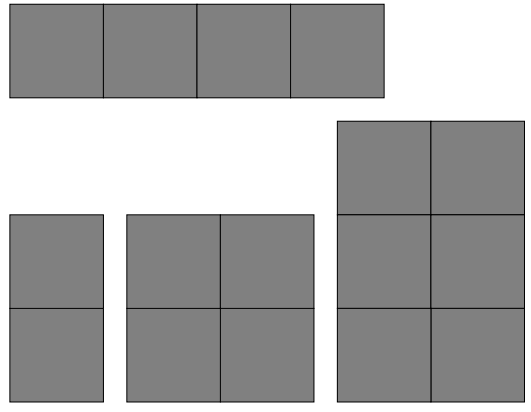

Set of 4 items

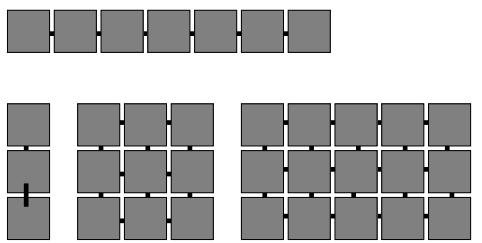

Pattern map composed of 4 components

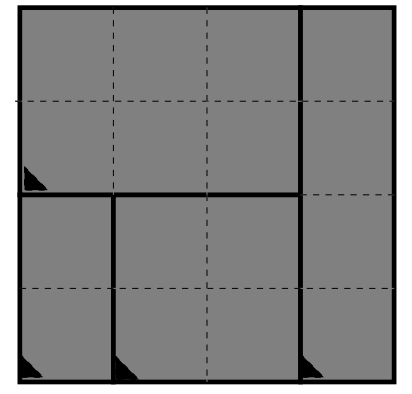

Solution for a bin of size $4 \times 4$

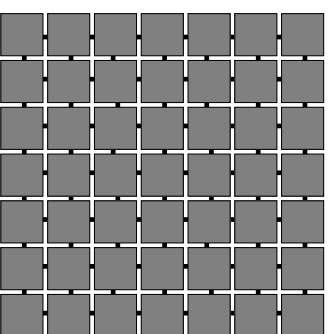

Target map

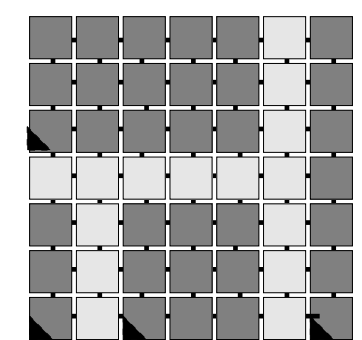

Solution of the submap isomorphism problem

Fig. 5. Instance of a strip packing problem (on the top) and its associated submap isomorphism problem (on the bottom): the strip packing instance is defined by 4 items of sizes $1 \times 2,2 \times 2,2 \times 3$, and $4 \times 1$, and a bin of size $4 \times 4$. To reduce this problem to a subisomorphism problem, we define a non connected $2 \mathrm{D}$ pattern map that is composed of 4 connected components respectively composed of $1 \times 3$, $3 \times 3,5 \times 3$, and $7 \times 1$ sewn squares, and we define a connected target map that is composed of $7 \times 7$ sewn squares. The solution to the strip packing problem is deduced from the solution to the submap isomorphism problem: the bottom left corner of the image of each connected component in the target map (marked by a black triangle) gives the position of the bottom left corner of the corresponding item in the bin (also marked by a black triangle).

a solution of the strip packing problem, this shows that submap isomorphism of non-connected maps is NP-complete.

\section{Constrained Isomorphisms}

In 2D image processing, the type of maps one wishes to consider have the property that they are to be drawn on the plane, not on the sphere. We call this type of map a planar combinatorial map. To understand this point, let us consider the typical situation represented in Fig. 6. These two maps are isomorphic on the sphere, which corresponds to the definitions introduced in Section 3.1, but are not isomorphic in the plane. In other words, for these two maps, Algorithm 3 returns true, but that is probably not what is intended in an image processing task. 


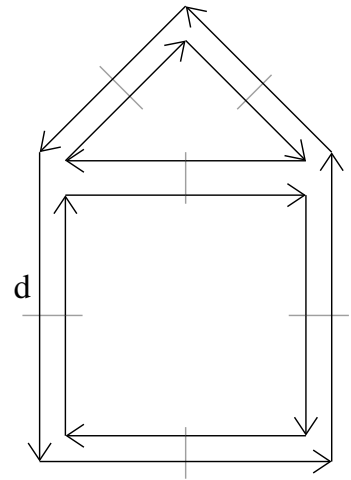

(a)

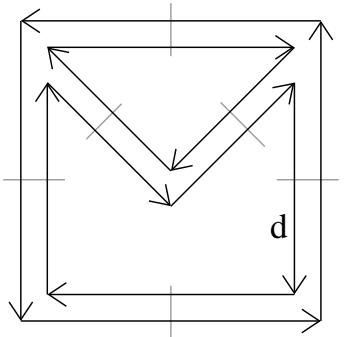

(b)

Fig. 6. Two maps that are isomorphic but not planar-isomorphic.

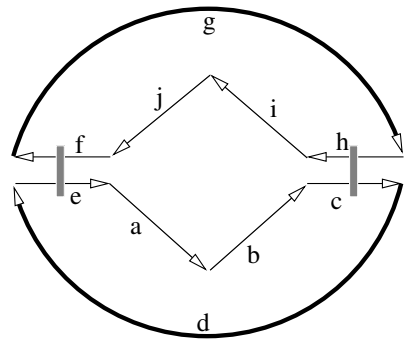

(a)

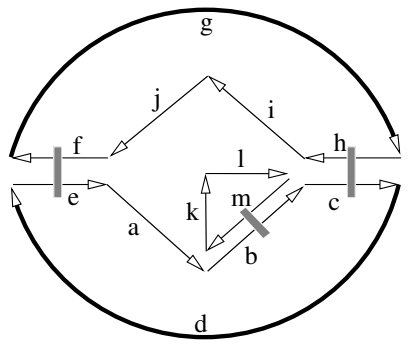

(b)

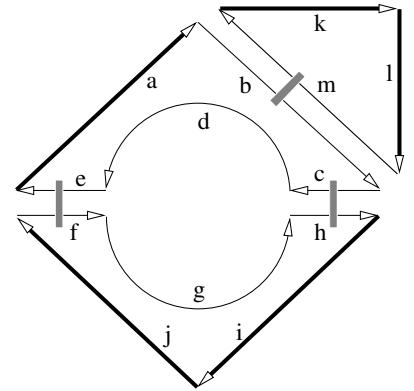

(c)

Fig. 7. Isomorphisms of maps with undefined parts on a sphere versus in the plane: on a sphere, (b) and (c) are isomorphic, and (a) is a submap of both (b) and (c). However, in the plane (b) and (c) are not isomorphic, and (a) is a submap of (b) but not of (c). Exterior darts are drawn in bold.

The property of being planar isomorphic is a geometrical property, i.e. it cannot be characterized for maps without geometry. Indeed, two maps are planar isomorphic when they are isomorphic and when the image of the exterior of the first map is equal to the exterior of the second map.

Let us consider, for example, the two maps displayed in Fig. 7(b) and Fig. 7(c). These maps are isomorphic but not planar isomorphic. Indeed, the image of the exterior darts of the map in Fig. 7(b) are not exterior darts in Fig. 7(c). This means that we have reversed the first map which is not a possible operation in the plane. This is similar for planar submap isomorphism. In our example, the map of Fig. 7(a) is a submap of the two maps of Fig. 7(b) and Fig. 7(c), but it is only a planar submap of the map of Fig. 7(b) since only in this case, the image of the exterior darts of Fig. 7(a) are still at the exterior of the second map.

In Section 4.1, we formally define planar maps by introducing the notion of exterior and infinite darts of a map. In Section 4.2, we extend (sub)map isomorphism to planar (sub)map isomorphism isomorphism by adding constraints on 


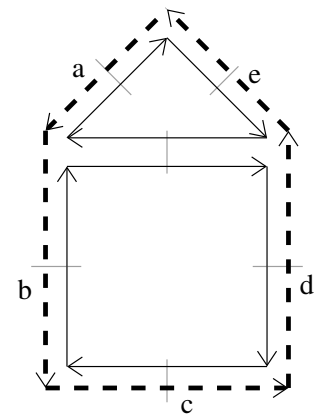

(a)

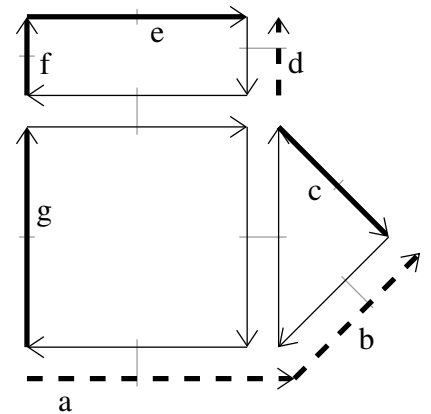

(b)

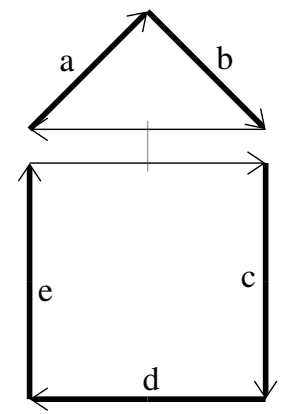

(c)

Fig. 8. Exteriors darts are drawn in bold, and infinite darts in dash. Darts in bold and dash are both exterior and infinite. (a) A closed map: Inf $=E x t=\{a, b, c, d, e\}$. (b) A map with an open infinite face: Inf $=\{a, b, d\}$ and $E x t=\{a, b, c, d, e, f, g\}$.

(c) A map without infinite face: Inf $=\emptyset$ and $E x t=\{a, b, c, d, e\}$.

exterior and infinite darts. In Section 4.3, we show how to compute exterior and infinite darts in the case of 1-closed combinatorial maps (i.e. when all faces are closed).

\subsection{Planar maps}

When considering a map drawn on the plane, a part of the plane corresponds to the exterior of the modeled object. There are 3 cases to consider:

- If there exists an infinite (or unbounded) face, and if this infinite face is closed, then the exterior is defined by the darts of this infinite face, as displayed in Fig. 8(a).

- If there exists an infinite face, but this face is open, then the exterior is defined by the darts of this open infinite face, plus the set of 2-free darts that border the exterior, as displayed in Fig. 8(b);

- If there does not exist an infinite face, then the exterior is defined by the set of 2-free darts that border the exterior, as displayed in Fig. 8(c).

Hence, a planar map is defined by a map, together with a set Ext of exterior darts, and a set Inf $\subseteq$ Ext of darts that belong to the infinite face (called infinite darts).

The last consideration to take into account is face orientation. Indeed, there are two possible orientations of each planar combinatorial map: by taking $\beta_{1}$ such that faces are oriented clockwise (like Fig. 9(b)) or counterclockwise (like Fig. 9(a)). Note that all the faces of a map have the same orientation, except the infinite face which has a reversed orientation. This orientation is once again a geometrical property, and it is required to use the same orientation for all the considered planar maps. Thus, in this section, we suppose that the 


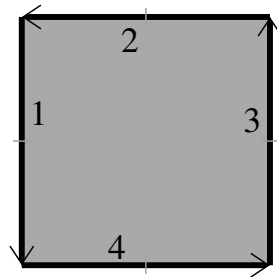

(a)

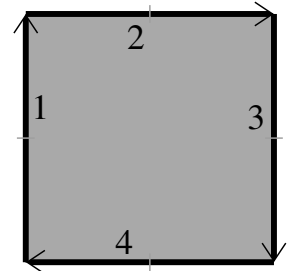

(b)

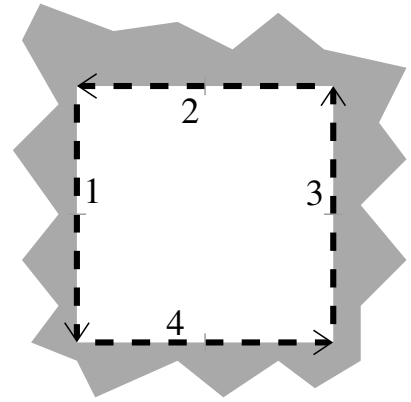

(c)

Fig. 9. Three maps which are isomorphic, but not planar isomorphic. (a) and (b) are two maps with different orientations. (b) and (c) have the same orientation but (b) has no infinite face while (c) has one.

same orientation is chosen for all the planar maps.

Definition 14 (Planar map) A planar map is a triple $P M=\langle M$, Ext, Inf $\rangle$ such that (1) $M=\left(D, \beta_{1}, \beta_{2}\right)$ is a combinatorial map drawn on the plane with a given orientation; (2) Ext $\subseteq D$ is the set of exterior darts; (3) Inf $\subseteq$ Ext is the set of infinite darts.

Note that since we only consider connected maps, there is at most one infinite face. This is not the case for non-connected maps that may have several infinite faces (at most one for each connected component).

\subsection{Planar map isomorphism}

When comparing two planar maps in order to decide if they are isomorphic, one has to check that the isomorphism function actually matches exterior and infinite darts, as stated in Def. 15.

Definition 15 (Planar map isomorphism) Two planar maps $P M=$ $\langle M$, Ext, Inf $\rangle$ and $P M^{\prime}=\left\langle M^{\prime}, E x t^{\prime}\right.$, In $\left.f^{\prime}\right\rangle$ are planar isomorphic if there exists an isomorphism function $f$ between $M$ and $M^{\prime}$ such that $f(E x t)=$ Ext $^{\prime}$ and $f(\operatorname{Inf})=\operatorname{In} f^{\prime}$.

The test on exterior darts is not enough to process all the possible cases. Let us consider for example the maps of Fig. 9(b) and Fig. 9(c). These two maps are isomorphic and have the same set of exterior darts, but they are not planar isomorphic since the first one represents a square face while the second one represents the plane minus a square. The condition on the infinite face allows to distinguish these two cases since $\operatorname{In} f=\emptyset$ in the first map, whereas $\operatorname{In} f \neq \emptyset$ in the second map.

The definition of a submap is also extended to planar maps as follows. 
Definition 16 (Planar submap) Given a planar map $P M=\langle M$, Ext, Inf $\rangle$ with $M=\left(D, \beta_{1}, \beta_{2}\right)$, and a subset of connected darts $X \subseteq D$. The planar submap of $P M$ induced by $X$ is the planar map $P M_{\downarrow X}=\left\langle M_{\downarrow X}, E x t^{\prime}\right.$, Inf $\left.^{\prime}\right\rangle$ such that $M_{\downarrow X}$ is the submap of $M$ induced by $X$, Ext ${ }^{\prime}$ is the set of exterior darts of $M_{\downarrow X}$ and Inf' is the set of infinite darts of $M_{\downarrow X}$.

The way to compute $E x t^{\prime}$ and $I n f^{\prime}$ depends on how the combinatorial map is linked with some geometrical elements. In particular, we show in Section 4.3 how these sets can be computed when combinatorial maps are 1-closed.

Planar submap isomorphism is now defined in a straightforward way with respect to planar submap and planar map isomorphism.

Definition 17 (Planar submap isomorphism) A planar map PM is planar submap isomorphic to a planar map $P M^{\prime}$ if there exists a planar submap of $P M^{\prime}$ which is planar isomorphic to $P M$.

\subsection{The case of 1-closed combinatorial maps}

In the field of image processing, faces are either entirely represented or not at all. Technically, this means that $\beta_{1}$ should be a permutation and not a partial permutation. We use this property in this section to simplify handling of exterior and infinite darts, and to show how to compute Ext and Inf sets for the planar submap definition.

If a map is 1-closed, we have only two cases to consider: the infinite face is either present and closed or totally absent. Therefore, we have either Inf $=\emptyset$, or Inf $=$ Ext. Thus, we do not have to explicitly list the set of infinite darts Inf, but we can simply use a boolean infinite which is true if the infinite face is present and false otherwise.

For each of these two cases, the subset Ext may be defined by giving only one dart $d_{\text {ext }}$ of the set:

- When the infinite face is defined, given a dart $d_{\text {ext }}$ belonging to this face, the whole set of exterior darts corresponds to the orbit $\left\langle\beta_{1}\right\rangle\left(d_{e x t}\right)$. For example, in Fig. 8(a), Ext $=\left\langle\beta_{1}\right\rangle(a)=\{a, b, c, d, e\}$.

- When the infinite face is not defined, the exterior darts correspond to the set of 2 -free darts that border the exterior of the map. In this case, given a 2-free dart $d_{e x t}$ that borders the exterior of the map, the whole set of exterior darts may be computed by Algorithm 4. For example, in Fig. 8(c), $E x t=\operatorname{BuILDEXTERIOR}(M, a$, false $)=\{a, b, c, d, e\}$.

Note that in both cases, there may exist 2-free darts that are not exterior 
darts. These 2-free darts border undefined parts of the map. Let us consider, for example, the map displayed in Fig. $7(\mathrm{a})$ : the set of exterior darts is $\{d, g\}$; the other 2 -free darts, i.e., $\{a, b, i, j\}$ border an undefined part inside the map.

Algorithm 4: BUILDEXTERIOR $\left(M, d_{\text {ext }}\right.$, infinite $)$

Input: a map $M=\left(D, \beta_{1}, \beta_{2}\right)$, an initial exterior dart $d_{e x t} \in D$, and a boolean infinite which is true if $d_{e x t}$ belongs to the infinite face, false otherwise

Output: the set of all exterior darts

1 if infinite is true then return $\left\langle\beta_{1}\right\rangle\left(d_{\text {ext }}\right)$

2 Ext $\leftarrow \emptyset ; d_{\text {curr }} \leftarrow d_{\text {ext }}$

3 repeat

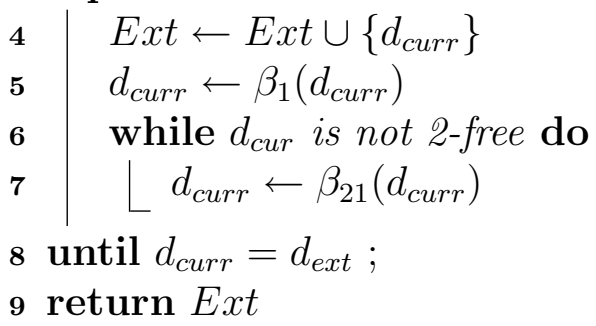

Theorem 18 Given $\left(M, d_{\text {ext }}\right.$, infinite), BUILDEXTERIOR computes all the exterior darts of $M$

PROOF. As the map is 1-closed, there are only two possible cases: the infinite face is present and thus Ext $=\operatorname{Inf}=\left\langle\beta_{1}\right\rangle\left(d_{e x t}\right)$, or this face is not present and in this case, the exterior darts can all be reached starting from $d_{\text {ext }}$, and keeping all the 2 -free darts that border the exterior of the map. For that, given an exterior dart, we go to the next dart of the same face (by using $\beta_{1}\left(d_{\text {curr }}\right)$ ), then we jump over darts inside the map (i.e. which are not 2 -free) by using $\beta_{21}\left(d_{\text {curr }}\right)$ as many time as necessary to reach a 2 -free dart. We are sure that such a dart exists because the map is connected and 1-closed.

This algorithm has a linear time complexity with respect to the number of darts.

We can now simplify the notation of planar maps by $P M=\left\langle M, d_{\text {ext }}\right.$, infinite $\rangle$ since we can retrieve Ext and Inf given $d_{e x t}$ and infinite, for 1-closed maps.

Note that two planar maps may be planar isomorphic only if the two sets of exterior darts have the same cardinality and either both correspond to an infinite face, or both correspond to a set of 2 -free darts that border the exterior of the maps. If this is not the case, one can trivially conclude that the two planar maps are not isomorphic; otherwise, one has to search for an isomorphism function that matches Ext and Ext'. In this later case, one may 
use the fact that darts of Ext must be matched to darts of Ext' to boost the research, as described in Algorithm 5.

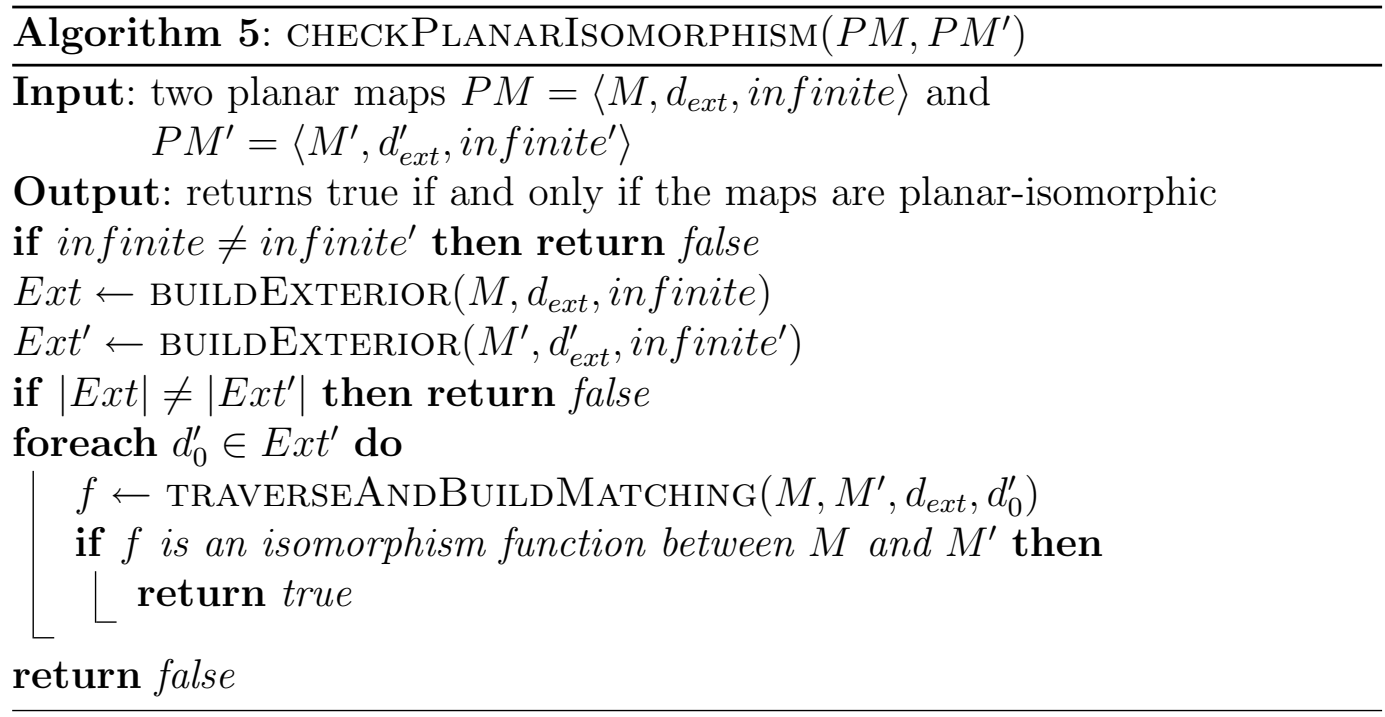

Theorem 19 CHECKPlanARIsOmorphism $\left(P M, P M^{\prime}\right)$ returns true iff $P M$ and $P M^{\prime}$ are planar isomorphic.

PROOF. First, if $P M$ and $P M^{\prime}$ are planar isomorphic, there is an isomorphism function $f$ such that $f(E x t)=E x t^{\prime}$ and $f(\operatorname{In} f)=I n f^{\prime}$. Thus, we have necessarily infinite $=$ infinite ${ }^{\prime}$ and $f\left(d_{\text {ext }}\right)=d_{0}^{\prime} \in E x t^{\prime}$. By testing all the darts $d_{0}^{\prime} \in E x t^{\prime}$, we are sure to find this case and thus the algorithm will return true. Second, if our algorithm returns true, we have an isomorphism function between $M$ and $M^{\prime}$ such that infinite $=$ infinite ${ }^{\prime}$ and $f\left(d_{\text {ext }}\right)=d_{0}^{\prime} \in$ Ext $^{\prime}$. Since we are in the case of 1 -closed maps, this implies that $f(\operatorname{Inf})=\operatorname{In} f^{\prime}$ and $f(E x t)=E x t^{\prime}$ and thus $P M$ and $P M^{\prime}$ are planar isomorphic.

Remember that we are able to decide of the isomorphism of 2-maps in $\mathcal{O}(|D|$. $\left.\left|D^{\prime}\right|\right)$ time. Having an information about the set of exterior darts and an efficient way to compute these darts allows us to reduce the complexity to $\mathcal{O}\left(\left|E x t^{\prime}\right| \cdot|D|\right)$. In many cases, $\left|E x t^{\prime}\right|$ is significantly smaller than $\left|D^{\prime}\right|$. Typically, $\left|E x t^{\prime}\right|=\mathcal{O}\left(\sqrt{\left|D^{\prime}\right|}\right)$ for the lattice $\mathbb{Z}^{2}$.

Now let us re-consider the planar submap problem. By using the fact that maps are 1-closed, we are now able to compute combinatorially Ext' and Inf $f^{\prime}$ in the submap definition. First, we need to add a constraint in the submap definition. Indeed, given a planar map $P M=(M, E x t$, Inf $)$, the subset of darts $X \subseteq D$ must ensure that the submap $P M_{\downarrow X}$ is always 1-closed. For this reason, for each face $f$ of the initial map, $X$ must either contain all the darts 
of $f$, or no dart of $f$, but it cannot contain only some darts of $f$, otherwise some darts are 1-free.

If the submap is 1-closed, then the infinite face is either totally kept in the submap, or totally removed. This allows us to compute simply the new boolean

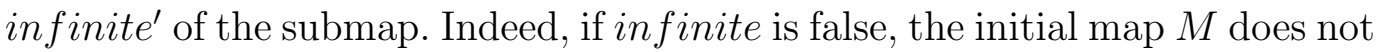
have an infinite face, and thus the submap also. Otherwise, we have Ext $=\operatorname{Inf}$ and thus $d_{e x t} \in \operatorname{Inf}$. Now there are again two cases: if $d_{e x t} \in X$, then the infinite face is totally selected and thus is present in the submap, otherwise the infinite face is not selected and thus the submap does not have an infinite face.

For $d_{\text {ext }}^{\prime}$, there are also two cases to consider. If $d_{e x t} \in X$, then this dart is present in the submap and thus it belongs to the exterior of the submap, so that $d_{e x t}^{\prime}=d_{e x t}$. Otherwise, we need to find a new exterior dart of the submap. This can be achieved easily by searching a dart $d \in X$ which can be reached from $d_{\text {ext }}$ by a path of darts that uses only darts that do not belong to $X$ (except $d$ of course). Indeed, such a dart belongs necessarily to the exterior of the submap induced from $X$ due to the fact that maps are connected. Such a path may be found by a simple traversal of the darts of $D$ starting from $d_{\text {ext }}$ and avoiding darts of $X$. The key point is to ensure that we are always able to compute the dart $d_{e x t}^{\prime}$ by using such a path. Suppose it is not the case, then there is no path between $d_{\text {ext }}$ and one dart of $X$ and this implies that the map is not connected.

More formally, we define planar submap for 1-closed maps as follows.

Definition 20 (1-closed planar submap) Given a 1-closed planar map $P M=\left\langle M, d_{\text {ext }}\right.$, infinite $\rangle$ with $M=\left(D, \beta_{1}, \beta_{2}\right)$, and a subset of connected darts $X \subseteq D$. The planar submap of $P M$ induced by $X$ is the planar map $P M_{\downarrow X}=\left\langle M_{\downarrow X}, d_{\text {ext' }}\right.$, infinite $\rangle$ such that $M_{\downarrow X}$ is 1-closed, with:

- infinite $^{\prime}=$ true if infinite $=$ true and $d_{\text {ext }} \in X$; infinite $^{\prime}=$ false otherwise;

- $d_{e x t}^{\prime}=d_{e x t}$ if $d_{e x t} \in X$; $d_{\text {ext }}^{\prime}=d \in X$ with a path $\left(d_{\text {ext }}, \ldots, d\right)$ of darts $\notin X$ (except $\left.d\right)$ otherwise.

Algorithm 6 shows how to decide if a planar map is isomorphic to a submap of another planar map. The basic idea is to compute subisomorphism functions and check if they satisfy the additional planarity constraint. We may have to compute several subisomorphism functions since it is possible that there are different subisomorphisms, and that some of them are planar while others are not.

Theorem 21 CheckPlanarSubIsomorphism $\left(P M, P M^{\prime}\right)$ returns true iff $P M$ is planar isomorphic to a submap of $P M^{\prime}$. 


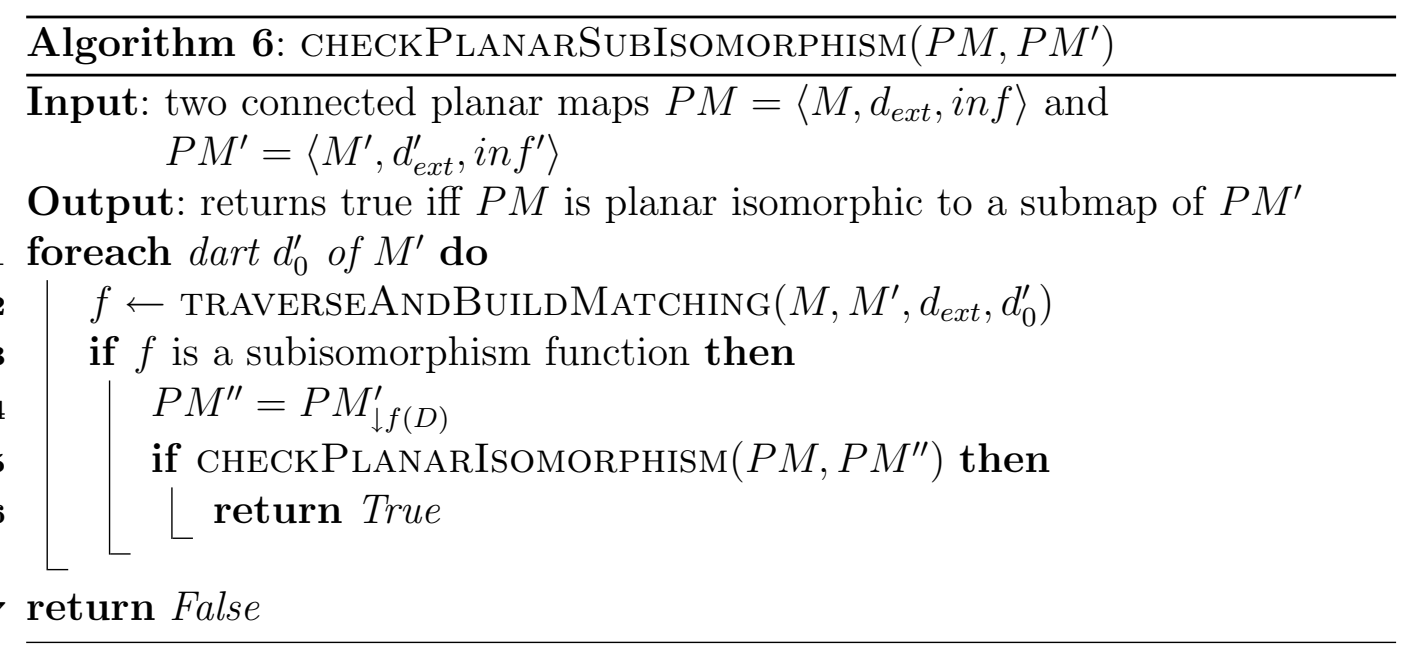

PROOF. First, if $P M$ is planar isomorphic to a submap of $P M^{\prime}$, as we compute all the subisomorphism functions between $M$ and $M^{\prime}$, we are sure to find the one satisfying planarity constraints and thus the algorithm returns true. Second, if our algorithm returns true, there is a subisomorphism satisfying the planarity constraints and thus $P M$ is planar isomorphic to a submap of $P M^{\prime}$.

The complexity of the planar subisomorphism algorithm is $\mathcal{O}\left(|D|^{2} \cdot\left|D^{\prime}\right|\right)$.

One loop of this algorithm is done by using Algorithm 2, which complexity (in 2D) is $\mathcal{O}(|D|)$. The complexity of planar submap computation is $\mathcal{O}\left(\left|D^{\prime}\right|\right)$, thanks to the efficient computation of infinite ${ }^{\prime \prime}$ and $d_{\text {ext }}^{\prime \prime}$. Finally the test of planar isomorphism is achieved by using Algorithm 5, the complexity of which is $\mathcal{O}\left(\left|E x t^{\prime \prime}\right| \cdot|D|\right)$. Since the number of darts in $E x t^{\prime \prime}$ is smaller than the number of darts in $D^{\prime \prime}$, we can bound the overall complexity of one loop by $\mathcal{O}\left(|D|^{2}\right)$ since $|D|=\left|D^{\prime \prime}\right|$.

\section{$5 \quad$ Experiments}

In this section, we carry out two experiments that show the effectiveness of our algorithms to detect patterns in images. The first challenge consists in searching for a sub-image into a database of 2D segmented images - a typical "Where's Wally" challenge. The second one aims at retrieving a sub-mesh into a database of $3 \mathrm{D}$ objects.

These experiments illustrate the interest of generic algorithms for submap isomorphism. Indeed, the same procedure is used whatever the dimension of the maps that model objects (2D, 3D or more). Moreover, we observe that the 


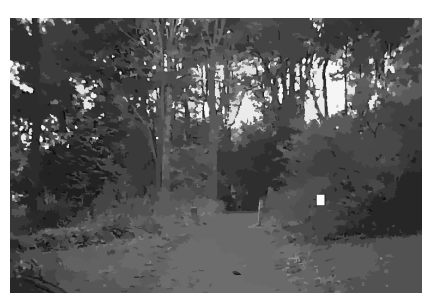

(a)

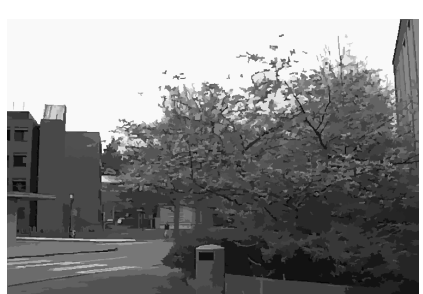

(b)

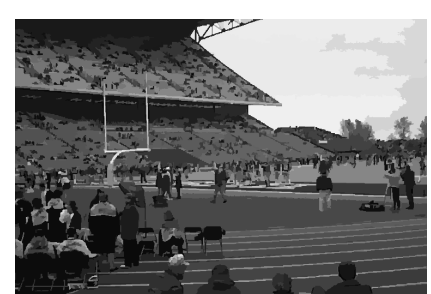

(c)

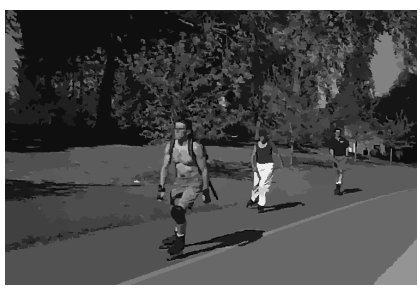

(d)

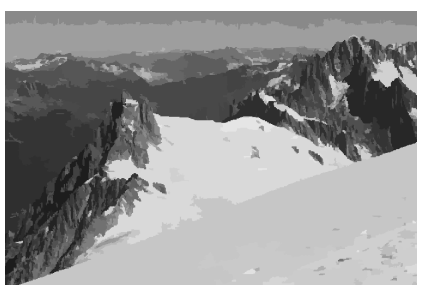

(e)

Fig. 10. Examples of 2D segmented images. All images have the same size $(756 \times 504$ pixels) and belong to one of the five following classes: (a) ARBOGREENS, (b) CHERRies, (c) Football, (d) GREEnlake and (e) SWissmountains.

time required to find patterns is always challenging. All our experiments were made on a PC with a $2.26 \mathrm{GHz}$ Intel Xeon E5520 processor.

\section{$5.12 D$ sub-image searching}

We first consider a database containing 224 segmented $2 \mathrm{D}$ images ${ }^{5}$ divided into 5 classes of approximately 45 images each: ARBORGREENS, CHERRIES, FOOTBALl, GREENLAKE and SWissmountains (see Fig. 10).

From these 224 images, we have randomly chosen 8 images from the database, and manually selected the 8 sub-images (SIm1 to SIm8) that are displayed in Fig. 11. The aim of this first experiment is to retrieve the images from which these sub-images were extracted.

First of all, we have computed the 2D planar maps of all the images of the database and of the 8 sub-images. To achieve this task, we used the algorithm of Damiand, Bertrand and Fiorio presented in [DBF04]. We describe in Table 1 the characteristics of the maps that correspond to the selected sub-images, as well as the average characteristics of the images of the database.

Let us first note that several maps are not connected. In this case, we cannot directly use our algorithm since it assumes that the pattern map is connected. To get round this problem, we have only kept the largest connected component

\footnotetext{
5 The database of free images for research purpose is available on Internet http://www.cs.washington.edu/research/imagedatabase/
} 


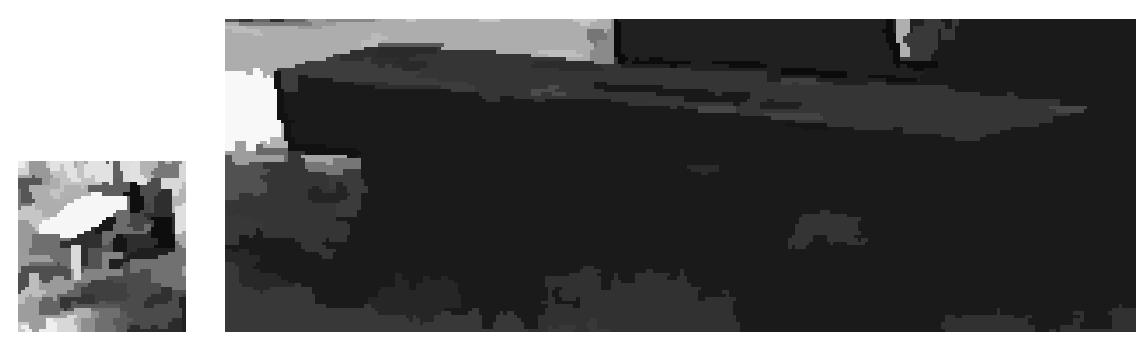

(a)

(b)

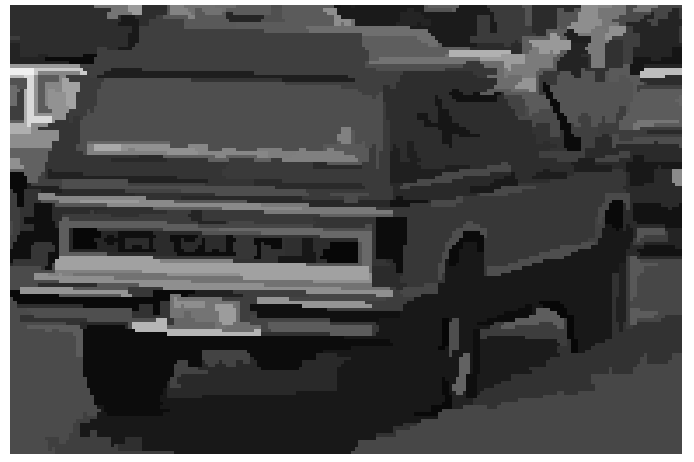

(c)

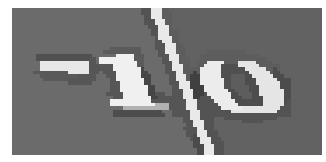

(f)

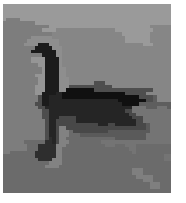

$(\mathrm{g})$

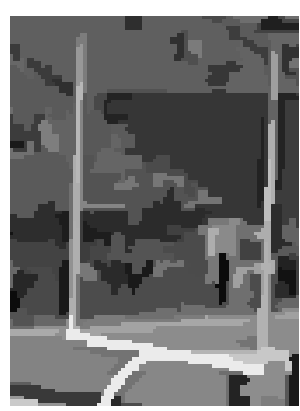

(d)

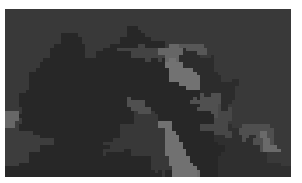

(h)

Fig. 11. The 8 selected sub-images. (a) SIm1, from arbogreens05, size $48 \times 49$ pixels. (b) SIm2, from arbogreens27, size $262 \times 90$ pixels. (c) SIm3, from cherries14, size $193 \times 129$ pixels. (d) SIm4, from football05, size $83 \times 112$ pixels. (e) SIm5, from football11, size $25 \times 25$ pixels. (f) SIm6, from football16, size $89 \times 42$ pixels. (g) SIm7, from greenlake06, size $48 \times 54$ pixels. (g) SIm8, from swissmountains22, size $82 \times 48$ pixels.

of each combinatorial map. This can be done by a linear traversal of the darts of the map. Of course, it may happen that a map contains a copy of this connected component, but not the whole pattern. However, experiments have shown us that looking for the largest connected component is discriminant enough: in our test suite, it never happens that a map contains a copy of a largest connected component which comes from another image.

We present in Table 2 the results of the search of the images from which each sub-image was extracted, using our subisomorphism procedure. These results are described in terms of the time (in milliseconds) and of the number of darts visited during a traversal (note that $n$ traversals are required to decide if there is a copy of a pattern map in a target map that has $n$ darts).

Let us first note that images are processed very quickly: each image is processed in 26 milliseconds, on average, so that a subimage is found in 6 seconds 


\begin{tabular}{|c|r|r|r|r|r||r|}
\hline \hline Object & \# Darts & \# Vertices & \# Edges & \# Faces & \# CC & \# dBCC \\
\hline \hline SIm1 & 412 & 136 & 206 & 72 & 1 & 146 \\
\hline SIm2 & 398 & 134 & 199 & 81 & 8 & 108 \\
\hline SIm3 & 1542 & 509 & 771 & 272 & 5 & 956 \\
\hline SIm4 & 766 & 253 & 383 & 136 & 3 & 372 \\
\hline SIm5 & 48 & 16 & 24 & 14 & 3 & 30 \\
\hline SIm6 & 138 & 46 & 69 & 25 & 1 & 45 \\
\hline SIm7 & 100 & 33 & 50 & 21 & 2 & 50 \\
\hline SIm8 & 108 & 36 & 54 & 20 & 1 & 30 \\
\hline \hline DB (avg) & 14509 & 4789 & 7255 & 2598 & 66 & - \\
\hline
\end{tabular}

Table 1

Characteristics of the maps. The first 8 lines correspond to the 8 sub-images; the last line corresponds to the images of the database (average characteristics). Each line successively gives the number of darts, vertices, edges, faces, connected components, and number of darts of the largest connected component.

\begin{tabular}{|c|c|c|c|c|c|c|c|c|}
\hline & SIm1 & SIm2 & SIm3 & SIm4 & SIm5 & SIm6 & SIm7 & SIm8 \\
\hline \hline Average time & 32.8 & 25.2 & 32.9 & 28.8 & 24.7 & 21.8 & 21.8 & 24 \\
Max time & 80 & 64 & 88 & 76 & 64 & 56 & 56 & 60 \\
Std Dev & 15.9 & 11.6 & 15.7 & 13.7 & 11.9 & 10.1 & 10.1 & 11.2 \\
\hline Total time & 7304 & 5608 & 7308 & 6400 & 5496 & 4840 & 4840 & 5328 \\
\hline \hline Average \# darts & 56.4 & 24 & 47.7 & 21.9 & 23.1 & 31.8 & 31.3 & 24.8 \\
Min \# darts & 39 & 18 & 25 & 16 & 19 & 31 & 28 & 17 \\
Max \# darts & 146 & 108 & 956 & 372 & 30 & 45 & 50 & 30 \\
Std Dev & 8.3 & 6.5 & 61.5 & 23.6 & 2.2 & 1.4 & 2.1 & 1.7 \\
\hline
\end{tabular}

Table 2
Experimental results of the search of a sub-image in a base of $2 \mathrm{D}$ images. The first 4 lines give the time in milliseconds: average, maximum and standard deviation of the time to search for a sub-image in an image, followed by the total time of the search in the whole base of 224 images. The last 4 lines give the number of darts visited during a map traversal (average, minimum, maximum, and standard deviation). Note that the maximum is always reached for the image from which the sub-image was extracted. 
or so in the whole database of 224 images. Actually, the number of darts visited during the search process is usually far below the theoretical bound. Let us consider, for example, results for SIm3, that has 956 darts: the average number of visited darts for deciding if SIm3 belongs to an image is 47.7. This comes from the fact that we consider an optimized version of the algorithm which checks that the matching $f$ is an injection during the traversal, and which stops the traversal as soon as two different pattern darts are matched to a same target dart. Hence, on average, the algorithm visits 32 darts or so whereas the submaps have 217 darts or so. Note that, for each submap, the maximal number of visited darts is always equal to the number of darts of this submap: this maximal bound is reached once, when the submap algorithm returns true.

When the subimage is modeled by a non connected map, we only search for the largest connected component. In all our experiments, this allows us to retrieve only one image, from which the subimage has been actually extracted. Note that, in some cases, the number of darts of the largest connected component is rather small (e.g. 30 darts for SIm5 and SIm8). This shows us that the topology of a very small part of a map is discriminant enough to characterize the sub-image.

\subsection{D sub-mesh searching}

In our second experiment, we consider 3D objects, modelled by 3D maps, in order to illustrate the genericity of our algorithms. We consider a database of 800 objects $^{6}$ represented in 3D such as the four ones displayed in Fig. 12.

From these objects, we have extracted the 8 sub-objects (SMh1 to SMh8) displayed in Fig. 13. The characteristics of these 8 sub-objects, as well as the average characteristics of the meshes of the database, are given in Table 3.

As for the previous experiments, these maps are not necessarily connected. In this case, we select for each pattern the largest connected component and we use this connected component to search for the pattern. Like in the $2 \mathrm{D}$ case, if it may be possible that a map contains a copy of this connected component but not the whole pattern, experimental results have shown us that the largest connected component is discriminant enough: for the 800 objects of the database (which model very similar objects in some cases) we always find only one matching which corresponds to the object from which the sub-mesh was extracted.

\footnotetext{
6 These objects are available in the Shape Retrieval Contest web page http://www . aimatshape.net/event/SHREC/
} 


\begin{tabular}{|c|r|r|r|r|r|r||r|}
\hline \hline Object & \# Darts & \# vertices & \# edges & \# faces & \# volumes & \# cc & \# dBCC \\
\hline \hline SMh1 & 36624 & 1772 & 4813 & 3052 & 25 & 13 & 23208 \\
\hline SMh2 & 3311 & 155 & 418 & 276 & 14 & 7 & 1056 \\
\hline SMh3 & 60216 & 2753 & 7775 & 5018 & 12 & 6 & 29040 \\
\hline SMh4 & 198936 & 8717 & 23555 & 16578 & 1140 & 442 & 15480 \\
\hline SMh5 & 59040 & 2495 & 7410 & 4920 & 6 & 3 & 31512 \\
\hline SMh6 & 54252 & 2538 & 7097 & 4521 & 28 & 15 & 4380 \\
\hline SMh7 & 45756 & 2015 & 5824 & 3813 & 8 & 4 & 28416 \\
\hline SMh8 & 7860 & 335 & 985 & 655 & 6 & 3 & 6384 \\
\hline \hline DB & 179123 & 10652 & 24440 & 14927 & 2113 & 1018 & - \\
\hline
\end{tabular}

Table 3

Characteristics of the meshes. The first 8 lines correspond to the 8 sub-meshes; the last line corresponds to the meshes of the database (average characteristics). Each line successively gives the number of darts, vertices, edges, faces, volumes, connected components, and number of darts of the largest connected component.

\begin{tabular}{|c|c|c|c|c|c|c|c|c|}
\hline \hline & SMh1 & SMh2 & SMh3 & SMh4 & SMh5 & SMh6 & SMh7 & SMh8 \\
\hline Average time & 1.31 & 0.51 & 0.68 & 0.32 & 1.24 & 1.21 & 0.63 & 0.57 \\
Max time & 13.56 & 4.89 & 11.76 & 7.16 & 13.98 & 13.84 & 6.30 & 6.39 \\
Std Dev & 1.89 & 0.65 & 1.10 & 0.44 & 1.82 & 1.73 & 0.82 & 0.73 \\
\hline Total time & 1043 & 407 & 542 & 252 & 987 & 964 & 501 & 453 \\
\hline \hline Average \# darts & 187.19 & 119.25 & 297.31 & 65.99 & 270.44 & 135.16 & 341.39 & 108.21 \\
Min \# darts & 1 & 1 & 1 & 1 & 1 & 1 & 7 & 3 \\
Max \# darts & 23208 & 1056 & 29040 & 15480 & 31512 & 4380 & 28416 & 6384 \\
Std Dev & 816.67 & 90.15 & 1026.47 & 546.04 & 1123.02 & 159.14 & 1001.47 & 229.57 \\
\hline \hline
\end{tabular}

\section{Table 4}

Experimental results of the search of a sub-mesh in a base of 3D meshes. The first 4 lines give the time in seconds: average, maximum and standard deviation of the time to search for a sub-mesh in a mesh, followed by the total time of the search in the whole base of 800 meshes. The last 4 lines give the number of darts visited for each map traversal (average, minimum, maximum, and standard deviation). Note that the maximum is always reached for the mesh from which the sub-mesh was extracted. 


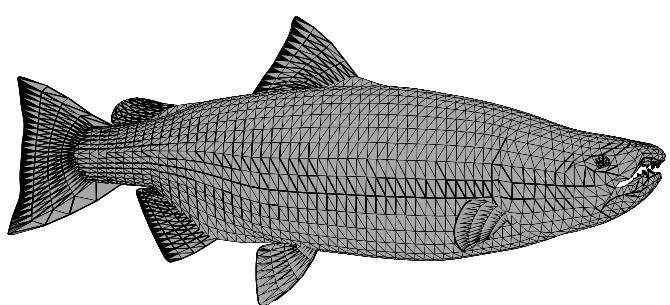

(a)

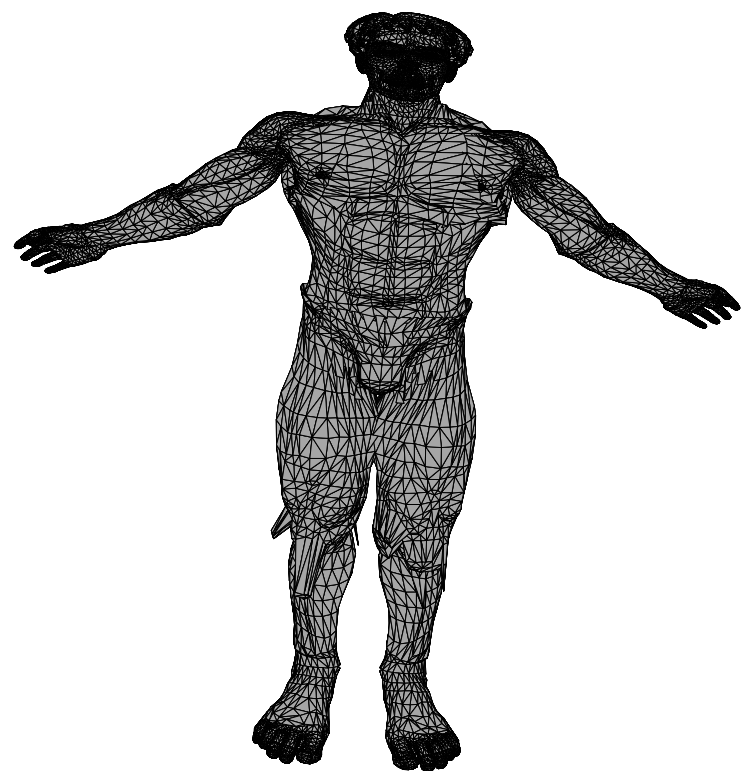

(c)

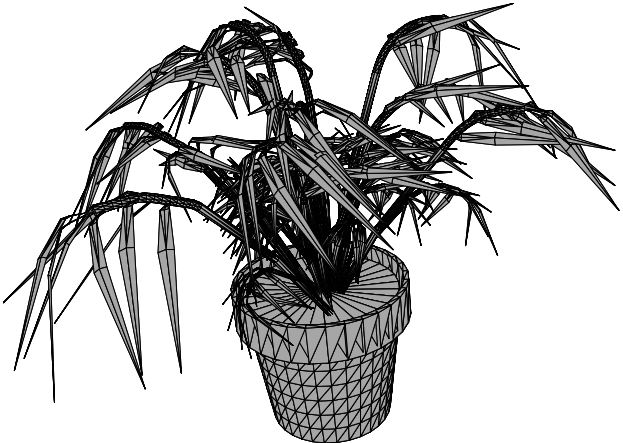

(b)

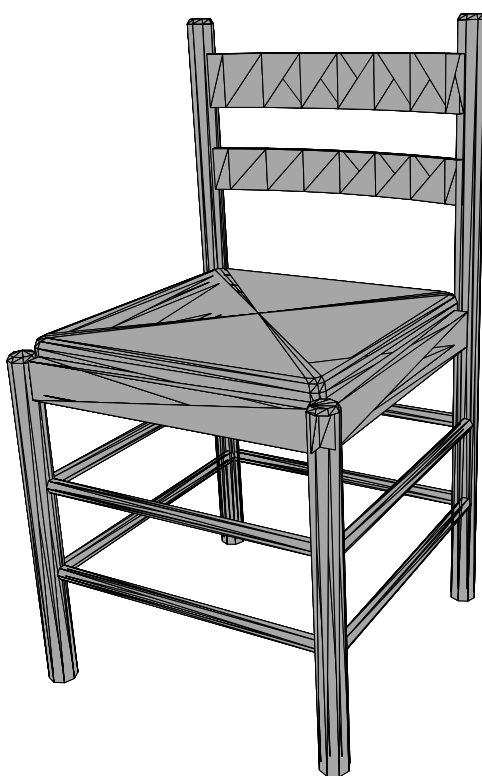

(d)

Fig. 12. Examples of 3D objects.

The results of our experiments are given in Table 4. Like in the 2D case, the number of visited darts during each traversal is often far below the worst case: the average number of visited darts is 190 whereas the sub-meshes have 17, 435 darts on average. Actually, in some cases only one dart is visited. This case occurs when the chosen initial pattern dart is free for one permutation whereas the chosen initial target dart is not free for this permutation. Hence, CPU times are still rather small when considering the fact that the database contains 800 meshes that have 180,000 darts or so on average (the largest meshes have more than one million darts). Indeed, the highest time needed to decide if a sub-mesh belongs to a mesh is smaller than 14 seconds, and the highest time needed to find a sub-mesh in the whole database of 800 meshes is 1043 seconds.

In Fig. 14, we can identify more precisely the correlation between the time needed by the submap algorithm and the size of the map in which we search the sub-mesh. Lines are linear regressions and show the difference of the slopes 


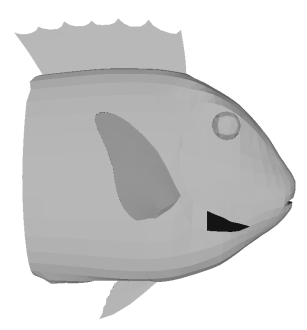

(a)

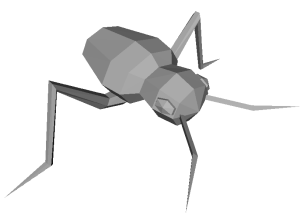

(b)

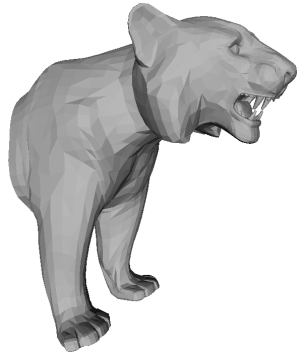

(c)

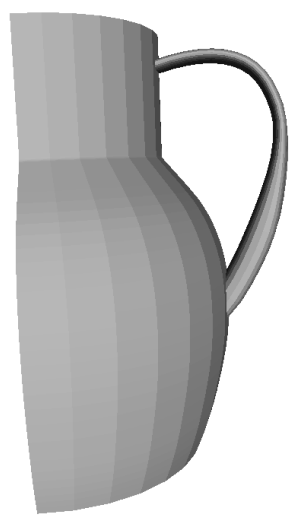

(g)

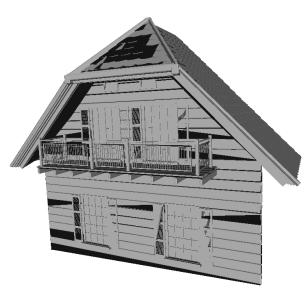

(d)

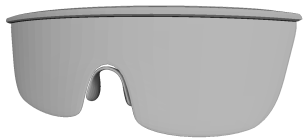

(e)

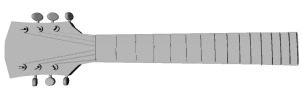

(f)

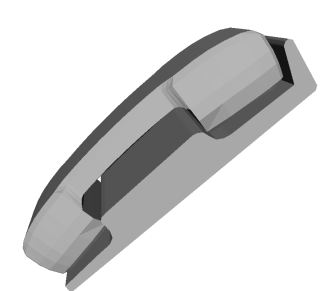

(h)

Fig. 13. The 8 selected sub-meshes.

between the different considered sub-meshes. In this figure, we can see a strong correlation between the number of darts of each target map and the time required by the submap search. However, we do not have a direct link between the slope of the linear regression and the number of darts of the biggest connected component of the sub-mesh. For example, this number of darts is 23208 for SMh1 and 29040 for SMh3, but the slope associated to SMh1 is below the slope associated to SMh3. This can be explained since the time depends not only on the number of darts of the largest connected component, but also on the topology of this component, i.e. on the way the darts are linked together.

\section{Discussion}

This paper is a first contribution to the problem of comparing combinatorial maps. It defines the map and submap isomorphism problems, which may be used to decide if two maps are equivalent, or if a copy of a map is included in another map, and it describes polynomial algorithms for solving these problems. The proposed definitions and algorithms are generic and hold for any open $n \mathrm{D}$ combinatorial maps, that may be used to model $n \mathrm{D}$ objects with boundaries. We also introduce planar combinatorial maps, which may be used 


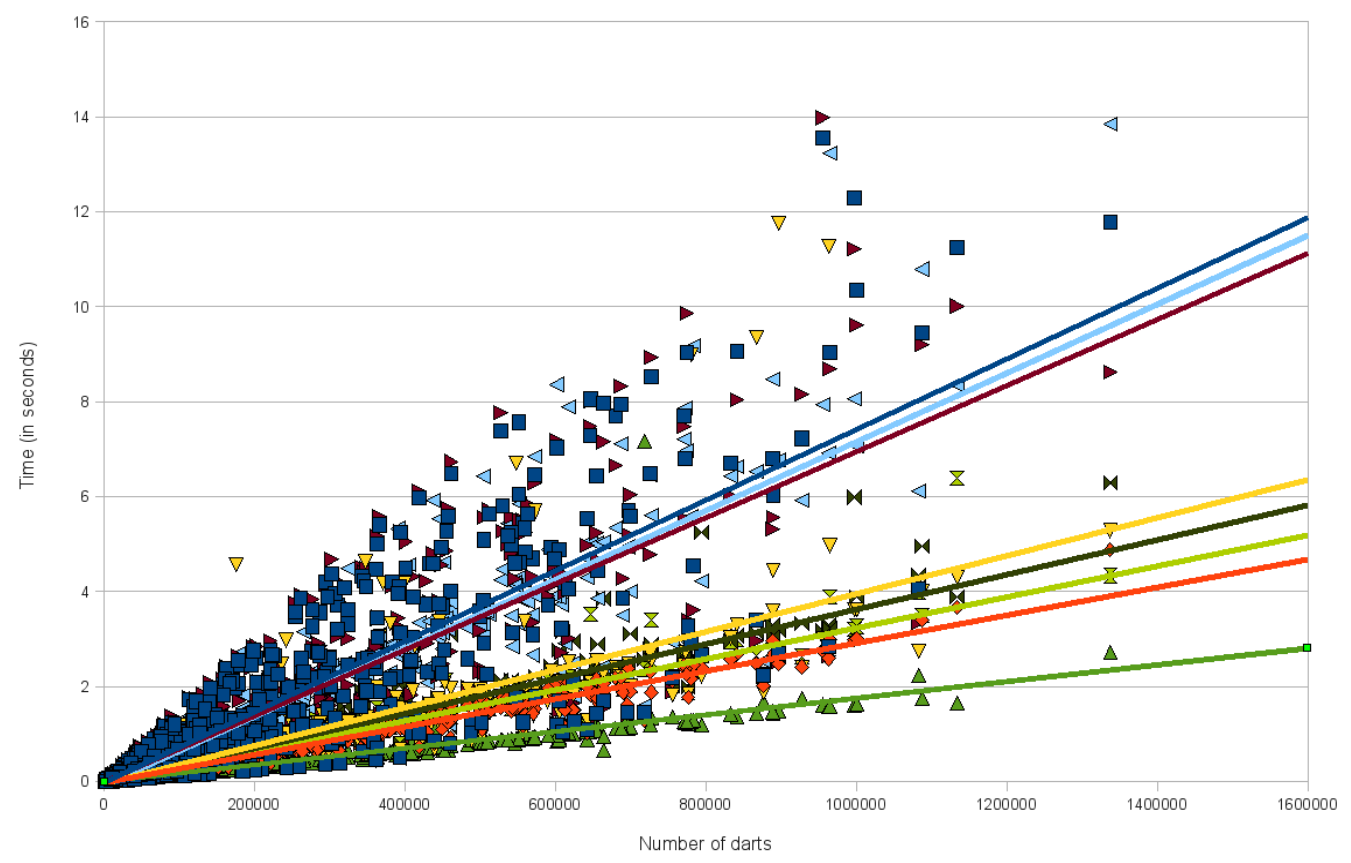

Fig. 14. Time in second to search each sub-mesh for each mesh of the database. SMh1 in dark blue, SMh2 in orange, SMh3 in yellow, SMh4 in green, SMh5 in dark red, SMh6 in light blue, SMh7 in dark green and SMh8 in light green.

to model 2D objects that are embedded on a plane such as, for example, images, and we show how to extend our algorithms to decide if two planar maps are isomorphic. Experiments show that this work may be used to search for patterns in 2D images or 3D meshes with very challenging $\mathrm{CPU}$ times.

The problem of finding a pattern in an image is an important issue that has often been tackled by modeling images with graphs such as, for example, RAGs. If there exist rather efficient heuristics for solving the graph isomorphism problem ${ }^{7}[\mathrm{McK} 81, \mathrm{SS} 08]$, this is not the case for the subgraph isomorphism problem which is computationally intractable in the general case (NP-complete), and therefore practically unsolvable for large scale graphs. In particular, the best performing approaches for subgraph isomorphism are usually limited to graphs up to a few hundreds of nodes [CFSV01,ZDS10].

Interestingly, using combinatorial maps to model images allows us to have more relevant results - as combinatorial maps model information that cannot be modelled with classical RAGs such as multi-adjacency or the order of neighbor regions around a region - and these results are computed much more quickly —as the subisomorphism problem becomes polynomial.

Some polynomial algorithms have been proposed to solve particular cases of

$\overline{7}$ The theoretical complexity of graph isomorphism is an open question: If it clearly belongs to NP, it has not been proven to be NP-complete. 
the subgraph isomorphism problem. In particular, Jiang and Bunke have proposed polynomial algorithms for deciding of the isomorphism [JB99] and subisomorphism [JB98] of ordered graphs, i.e., graphs such that, for each vertex, the edges incident to this vertex have a unique order. These algorithms are based on graph traversals and our algorithms for (sub)map isomorphism may be viewed as a generalization of this work to $n \mathrm{D}$ open combinatorial maps.

A number of perspectives are being looked into. In particular, (sub)map isomorphism is an exact decision problem which allows us to search for a pattern in a map. We plan to develop error-tolerant methods, that are able to quantify the similarity of two maps by means of the size of their largest common submap, or by means of the cost to transform a map into another by using the edit operations defined in [DL03,BADSM08]. We also plan to use our (sub)isomorphism algorithms to search for patterns that occur frequently in a database of images modelled by combinatorial maps, thus characterizing classes of images. We have proposed in [GDS09] a signature of combinatorial maps which is based on a map traversal similar to the one used in our isomorphism algorithm. This map signature may be used to decide in linear time if a new combinatorial map already belongs to a database of map signatures. We now plan to use this map signature and our submap algorithm in order to find frequent patterns in a database of maps.

\section{Acknowledgement}

The authors acknowledge authors of [PABL07], specially Pascal Lienhardt, for all the fruitful discussions about open combinatorial maps.

\section{References}

[AK89] D. Arques and P. Koch. Modélisation de solides par les pavages. In Proc. Pixim 89, pages 47-61, Paris, 1989.

[BADSM08] M. Baba-Ali, G. Damiand, X. Skapin, and D. Marcheix. Insertion and expansion operations for $\mathrm{n}$-dimensional generalized maps. In Proc. of 14th International Conference on Discrete Geometry for Computer Imagery (DGCI), volume 4992 of LNCS, pages 141-152, Lyon, France, April 2008. Springer-Verlag.

[BDB97] L. Brun, J.-P. Domenger, and J.-P. Braquelaire. Discrete maps : a framework for region segmentation algorithms. In Proc. Workshop on Graph-based Representations in Pattern Recognition, Lyon, april 1997. IAPR-TC15. published in Advances in Computing (Springer). 
[BDD01] J.-P. Braquelaire, P. Desbarats, and J.-P. Domenger. 3d split and merge with 3-maps. In Proc. Workshop on Graph-based Representations in Pattern Recognition, pages 32-43, Ischia, Italy, may 2001. IAPR-TC15.

[BDDW99] J.P. Braquelaire, P. Desbarats, J.P. Domenger, and C.A. Wüthrich. A topological structuring for aggregates of $3 \mathrm{~d}$ discrete objects. In Proc. Workshop on Graph-based Representations in Pattern Recognition, pages 193-202, Austria, may 1999. IAPR-TC15.

[Bru96] L. Brun. Segmentation d'images couleur à base Topologique. Thèse de doctorat, Université Bordeaux I, décembre 1996.

[CFSV01] L. P. Cordella, P. Foggia, C. Sansone, and M. Vento. An improved algorithm for matching large graphs. In Proc. Workshop on Graphbased Representations in Pattern Recognition, pages 149-159, Ischia, Italy, 2001.

[CFSV07] D Conte, P. Foggia, C. Sansone, and M. Vento. Applied Graph Theory in Computer Vision and Pattern Recognition, chapter How and Why Pattern Recognition and Computer Vision Applications Use Graphs, pages 85-135. Studies in Computational Intelligence. Springer Berlin / Heidelberg, 2007.

[Cor75] R. Cori. Un code pour les graphes planaires et ses applications. In Astérisque, volume 27. Soc. Math. de France, Paris, France, 1975.

[Dam08] G. Damiand. Topological model for 3d image representation: Definition and incremental extraction algorithm. Computer Vision and Image Understanding, 109(3):260-289, March 2008.

[DBF04] G. Damiand, Y. Bertrand, and C. Fiorio. Topological model for twodimensional image representation: definition and optimal extraction algorithm. Computer Vision and Image Understanding, 93(2):111154, February 2004.

[DD08] A. Dupas and G. Damiand. First results for 3d image segmentation with topological map. In Proc. Discrete Geometry for Computer Imagery, volume 4992 of Lecture Notes in Computer Science, pages 507-518, Lyon, France, April 2008. Springer-Verlag.

$\left[\mathrm{DDLHJ}^{+}\right.$09] G. Damiand, C. De La Higuera, J.-C. Janodet, E. Samuel, and C. Solnon. Polynomial algorithm for submap isomorphism: Application to searching patterns in images. In Proc. of 7th Workshop on Graph-Based Representation in Pattern Recognition (GBR), volume 5534 of LNCS, pages 102-112, Venice, Italy, May 2009. Springer-Verlag.

[DL03] G. Damiand and P. Lienhardt. Removal and contraction for n-dimensional generalized maps. In Proc. of 11th International Conference on Discrete Geometry for Computer Imagery (DGCI), 
volume 2886 of LNCS, pages 408-419, Naples, Italy, November 2003. Springer-Verlag.

[Dom92] J.P. Domenger. Conception et implémentation du noyeau graphique d'un environnement 2D1/2 d'édition d'images discrètes. Thèse de doctorat, Université Bordeaux I, avril 1992.

[DR02] G. Damiand and P. Resch. Topological map based algorithms for 3d image segmentation. In Proc. Discrete Geometry for Computer Imagery, number 2301 in Lecture Notes in Computer Science, pages 220-231, Bordeaux, France, april 2002.

[Edm60] J. Edmonds. A combinatorial representation for polyhedral surfaces. Notices of the American Mathematical Society, 7, 1960.

[Fio96] C. Fiorio. A topologically consistent representation for image analysis: the frontiers topological graph. In Proc. Discrete Geometry for Computer Imagery, number 1176 in Lecture Notes in Computer Science, pages 151-162, Lyon, France, november 1996.

[GDS09] S. Gosselin, G. Damiand, and C. Solnon. Signatures of combinatorial maps. In 13th Workshop on Combinatorial Image Analysis (IWCIA), volume 5852 of $L N C S$. Springer, 2009.

[GMBM95] T. Geraud, J.F. Mangin, I. Bloch, and H. Maitre. Segmenting internal structures in $3 \mathrm{~d} \mathrm{mr}$ images of the brain by markovian relaxation on a watershed based adjacency graph. In Proc. IEEE International Conference on Image Processing, volume 3, pages 548-551, oct 1995.

[JB93] X. Y. Jiang and H. Bunke. An optimal algorithm for extracting the regions of a plane graph. Pattern Recognition Letters, 14(7):553-558, 1993.

[JB98] X. Jiang and H. Bunke. Marked subgraph isomorphism of ordered graphs. In Proc. Advances in Pattern Recognition, volume 1451 of Lecture Notes in Computer Science, pages 122-131, 1998.

[JB99] X. Jiang and H. Bunke. Optimal quadratic-time isomorphism of ordered graphs. Pattern Recognition, 32(7):1273-1283, 1999.

[KM95] W.G. Kropatsch and H. Macho. Finding the structure of connected components using dual irregular pyramids. In Proc. Discrete Geometry for Computer Imagery, pages 147-158, invited lecture, september 1995.

[Kov89] V.A. Kovalevsky. Finite topology as applied to image analysis. Computer Vision, Graphics, and Image Processing, 46:141-161, 1989.

[Lie91] P. Lienhardt. Topological models for boundary representation: a comparison with n-dimensional generalized maps. Computer-Aided Design, 23(1):59-82, 1991. 
[Lie94] P. Lienhardt. N-dimensional generalized combinatorial maps and cellular quasi-manifolds. International Journal of Computational Geometry and Applications, 4(3):275-324, 1994.

[LMV01] J. Llados, E. Marti, and J.J. Villanueva. Symbol recognition by error-tolerant subgraph matching between region adjacency graphs. IEEE Transactions on Pattern Analysis and Machine Intelligence, 23(10):1137-1143, October 2001.

[McK81] B. D. McKay. Practical graph isomorphism. Congressus Numerantium, 30:45-87, 1981.

[PABL07] M. Poudret, A. Arnould, Y. Bertrand, and P. Lienhardt. Cartes combinatoires ouvertes. Research Notes 2007-1, Laboratoire SIC E.A. 4103, F-86962 Futuroscope Cedex - France, October 2007.

[Ros74] A. Rosenfeld. Adjacency in digital pictures. Information and Control, 26(1):24-33, 1974.

[Saa94] K. Saarinen. Color image segmentation by a watershed algorithm and region adjacency graph processing. In Proc. IEEE International Conference on Image Processing, volume 3, pages 1021-1025, nov 1994.

[SC84] M. Suk and T.H. Cho. An object-detection algorithm based on the region-adjacency graph. Proceedings of the IEEE, 72:985-986, 1984.

[Spe91] J.C. Spehner. Merging in maps and in pavings. Theoretical Computer Science, 86(2):205-232, September 1991.

[SS08] S. Sorlin and C. Solnon. A parametric filtering algorithm for the graph isomorphism problem. Constraints, 13(4):518-537, 2008.

[Tut63] W.T. Tutte. A census of planar maps. Canad. J. Math., 15:249-271, 1963.

[ZDS10] S. Zampelli, Y. Deville, and C. Solnon. Solving subgraph isomorphism problems with constraint programming. Constraints (to appear), 2010. 